Article

\title{
Novel Molecular Synapomorphies Demarcate Different Main Groups/Subgroups of Plasmodium and Piroplasmida Species Clarifying Their Evolutionary Relationships
}

\author{
Rahul Sharma and Radhey S. Gupta * \\ Department of Biochemistry and Biomedical Sciences, McMaster University, Hamilton, ON L8N 3Z5, Canada \\ * Correspondence: gupta@mcmaster.ca; Tel.: +(905)-525-9140 (ext. 22639)
}

Received: 16 May 2019; Accepted: 14 June 2019; Published: 28 June 2019

\begin{abstract}
The class Hematozoa encompasses several clinically important genera, including Plasmodium, whose members cause the major life-threating disease malaria. Hence, a good understanding of the interrelationships of organisms from this class and reliable means for distinguishing them are of much importance. This study reports comprehensive phylogenetic and comparative analyses on protein sequences on the genomes of 28 hematozoa species to understand their interrelationships. In addition to phylogenetic trees based on two large datasets of protein sequences, detailed comparative analyses were carried out on the genomes of hematozoa species to identify novel molecular synapomorphies consisting of conserved signature indels (CSIs) in protein sequences. These studies have identified 79 CSIs that are exclusively present in specific groups of Hematozoa/Plasmodium species, also supported by phylogenetic analysis, providing reliable means for the identification of these species groups and understanding their interrelationships. Of these CSIs, six CSIs are specifically shared by all hematozoa species, two CSIs serve to distinguish members of the order Piroplasmida, five CSIs are uniquely found in all Piroplasmida species except B. microti and two CSIs are specific for the genus Theileria. Additionally, we also describe 23 CSIs that are exclusively present in all genome-sequenced Plasmodium species and two, nine, ten and eight CSIs which are specific for members of the Plasmodium subgenera Haemamoeba, Laverania, Vinckeia and Plasmodium (excluding P. ovale and P. malariae), respectively. Additionally, our work has identified several CSIs that support species relationships which are not evident from phylogenetic analysis. Of these CSIs, one CSI supports the ancestral nature of the avian-Plasmodium species in comparison to the mammalian-infecting groups of Plasmodium species, four CSIs strongly support a specific relationship of species between the subgenera Plasmodium and Vinckeia and three CSIs each that reliably group P. malariae with members of the subgenus Plasmodium and P. ovale within the subgenus Vinckeia, respectively. These results provide a reliable framework for understanding the evolutionary relationships among the Plasmodium/Piroplasmida species. Further, in view of the exclusivity of the described molecular markers for the indicated groups of hematozoa species, particularly large numbers of unique characteristics that are specific for all Plasmodium species, they provide important molecular tools for biochemical/genetic studies and for developing novel diagnostics and therapeutics for these organisms.
\end{abstract}

Keywords: genome sequences; molecular markers (synapomorphies), phylogenetic trees; conserved signature indels; Hematozoa; Piroplasmida; evolutionary relationships among Plasmodium subgenera Haemamoeba; Laverania; Vinckeia and Plasmodium 


\section{Introduction}

The genus Plasmodium is comprised of eukaryotic unicellular parasites that parasitize a large variety of vertebrates [1-3]. Significantly, its members P. falciparum, P. vivax, P. knowlesi, P. ovale and P. malariae, are the causative agents of the major life-threating disease malaria [1,3]. The Babesia and Theileria genera, which are closely related to Plasmodium, are also of much interest and contain species that are clinically significant [4-6]. For example, Babesia microti is the primary causative agent of babesiosis which is a disease that manifests malaria-like symptoms in humans [6], whereas Theileria species are responsible for the disease theileriosis affecting domestic cattle causing significant economic losses within the farming industry [7]. The genera Babesia and Theileria form the majority of the named members within the order Piroplasmida [8-10] and this order along with the genus Plasmodium constitute the majority of species within the class Hematozoa (synonym Aconoidasida) in the phylum Apicomplexa [2,11,12]. In view of the enormous clinical significance and economic impact of the members of the class Hematozoa [12-14], it is of much importance to reliably understand the interrelationships of different parasitic organisms that are part of this class and develop reliable means for distinguishing them from each other.

The classification of species comprising the class Hematozoa was initially based on morphological characteristics such as the cellular ultrastructure, life-cycle details, and host range $[2,11,15]$. However, as most of these characteristics exhibit homoplasy, inferences based on them are considered unreliable [16-18]. In recent years, molecular sequenced data has been increasingly employed to understand hematozoa phylogeny $[3,8,10,12,16,19-22]$. In addition to the phylogenetic trees based on $18 \mathrm{~S}$ rRNA sequences, $c y t b$ gene sequences have been widely used for phylogenetic analysis and description of novel members of the class Hematozoa [23-26]. Contemporary work has expanded on this by using multigene phylogenetic trees for the study of Plasmodium $[12,16,19,27,28]$. This includes one of the most intensive analyses done on Plasmodium, utilizing 1000 genes shared amongst 12 Plasmodium species [29]. Similar approaches have also been applied for the study of Piroplasmida and also to a lesser extent on the class Hematozoa [8,10,30-32]. The results from these studies indicate that Piroplasmida and Plasmodium species are closely related and form two separate monophyletic clades. The two main genera of Piroplasmida (Babesia and Theileria) generally also form independent clades, except that $B$. microti, the primary agent of human babesiosis, branches distantly from other Babesia species $[5,10,33]$. These studies have also provided important insights into the interrelationships among the Plasmodium species. In general, the Plasmodium species parasitizing mammals versus those that parasitize other vertebrates (avian and lizard species) form two separate clades [16]. The latter group of species are placed into the subgenus Haemamoeba [34]. Within the mammalian-infecting species, 3 subgenera level groupings have been proposed [2,34-36]. Of these three subgroups, (i) subgenus Laverania contains P. falciparum and other great ape infecting species, (ii) subgenus Plasmodium includes P. vivax and other "Old World Monkey" infecting species, and (iii) subgenus Vinckeia is comprised of $P$. berghei and other non-primate infecting (rodent) species [36]. However, some human-infecting Plasmodium species e.g., P. ovale and P. malariae, do not consistently group with members of the subgenus Plasmodium and their phylogenetic placement remains uncertain [12,22,27-29,37]. Although recent phylogenetic studies have considerably advanced our understanding of the Plasmodium species, important questions remains concerning the interrelationships among different subgenera/groups within this genus [3,37-39]. Additionally, the genus Plasmodium and different subgroups within it are currently identified primarily on the basis of their branching in phylogenetic trees and the host specificity of the species, and no reliable molecular characteristics are known that are specific for these groups [3].

Genome sequences are currently available for large numbers of hematozoa species including 20 annotated genomes for Plasmodium species and 8 annotated genomes from the Babesia and Theileria genera. The available genomes provide a valuable resource for examining the evolutionary relationships amongst these species by construction of phylogenetic trees based on different datasets of genes/proteins sequences. More importantly, these genomes provide an extensive resource for comparative genomic 
studies for identifying novel molecular characteristics that are uniquely shared by members of the genera Plasmodium, Babesia and Theileria and could provide useful means for the demarcation of these taxa and for understanding their intra- and inter-relationships. One important class of molecular markers whose discovery has been facilitated by genome sequence analyses is comprised of conserved signature insertions/deletions (indels) (CSIs) in gene/protein sequences that are uniquely shared by an evolutionarily related group of species $[40,41]$. The CSIs that are useful for evolutionary studies are generally of definite lengths, present at specific positions in particular genes/proteins, and they are flanked on both sides by conserved regions to ensure that they constitute reliable characteristics [42-45]). The CSIs in genes/proteins sequences generally result from rare genetic changes and the most parsimonious explanation to account for their shared presence in a given gene or protein from a specific group of species is that the genetic change giving rise to the CSI first occurred in a common ancestor of the indicated group and then it was vertically inherited by the other group members [40,44-47]. Due to the discrete natures of the CSIs (even a one aa insertion or deletion in a protein sequence results from an in-frame three base pair insertion or deletion) and their location within conserved regions, their presence or absence in different lineages or proteins is generally not affected by factors such as differences in evolutionary rates among different species, or proteins, and long-branch attraction artefacts [40,41,45,48-50]. In view of these characteristics, the CSIs in gene/protein sequences have provided important means for demarcation of different groups of organisms in molecular terms [44,50]. Additionally, based upon the presence or absence of a CSI in outgroup species, it is possible to infer whether a given CSI represents an insert or a deletion in the protein sequence and thus infer the ancestral state of the protein. As a result, the CSIs in protein sequences can be used to infer rooted evolutionary relationships among a given group of species independently of the phylogenetic trees and they have proven very useful in clarifying a number of important relationships [40-43]. Although the shared presence of CSIs in protein sequences in some cases can result from homoplasy or lateral gene transfers [44,47,48], in general, when a conserved indel of a definite length is found uniquely in a phylogenetically related group of organisms, its most parsimonious explanation is inheritance from the most recent common ancestor $[40,44,51]$. Thus, the monophyletic group specific CSIs provide powerful means to support or refute a given phylogenetic hypothesis.

In the present study, we have used the available genome sequences to construct robust phylogenetic trees for hematozoa species based on two large datasets of concatenated protein sequences. In both trees, the Plasmodium species formed a strongly supported cluster that was separated from members of the order Piroplasmida by a long branch. Within the genus Plasmodium, monophyletic clades generally corresponding to the subgenera Laverania, Plasmodium, Vinckeia and Haemamoeba were also reliably observed. The trees also provide important information concerning the evolutionary relationships amongst the different Plasmodium species. Additionally, and more importantly, our comparative genomic analysis of the hematozoa genomes has identified 79 CSIs in different proteins that are uniquely shared by different members of this class. Of the identified molecular markers, 16 CSIs are specific for the class Hematozoa and either all or specific members of the order Piroplasmida. The remaining 63 CSIs are distinctive characteristics of either all 20 genome-sequenced Plasmodium species or specific subgroups/subgenera with the genus Plasmodium providing reliable molecular means for identification of these groups and clarifying their interrelationships. The described molecular markers in addition to their usefulness for evolutionary and taxonomic studies, due to their exclusivity for these clinically important groups of organisms, also provide potentially useful means for development of novel diagnostics and therapeutics that are specific for these organisms. 


\section{Materials and Methods}

\subsection{Construction of Phylogenetic Trees}

Phylogenetic trees were constructed for 28 genome sequenced members of the class Hematozoa and eight species from the order Eucoccidiorida, which were used for rooting of the trees. Some characteristics of the genome sequences that were used for tree construction are listed in Table S1. The phylogenetic trees were constructed based on two separate datasets of concatenated protein sequences, which are conserved in different hematozoa as well as the outgroup species. The first tree utilized 14 proteins which are involved in transcription and translation related functions, whereas the second tree was based on 10 metabolism-related proteins. These proteins were selected based on the criteria that they were within the majority of the members within the group of interest and that only a single homolog (or gene) of these proteins was detected in different hematozoa species. These selection criteria ensured that only orthologous sequences were used for the construction of the phylogenetic trees. Information for the proteins that were used for tree construction is provided in Tables S2 and S3, respectively. The phylogenetic tree construction was carried out using an internally developed pipeline that we have described in earlier work [46,52]. Briefly, the Clustal Omega algorithm was used to generate multiple sequence alignments for all proteins in a given dataset which are present in at least $80 \%$ of the input genomes. The aligned sequences were trimmed with TrimAl [53] to remove poorly aligned regions before they were concatenated into separate files. The final concatenated alignments for the transcription-translation related proteins and the metabolism-related datasets of protein sequences consisted of 7700 and 4068 aligned amino acid residues, respectively. Maximum-likelihood trees based on these alignments were constructed using FastTree 2 [54] and optimized using RAxML 8 [55] as described in earlier work [46].

\subsection{Identification of Conserved Signature Indels (CSIs)}

The identification of CSIs was carried out as described in earlier work [40,46,47]. In brief, BLASTp searches were carried out on all protein sequences from $P$. falciparum that were $>100$ amino acids in length, against the NCBI non-redundant database. Based on these searches, for those proteins for which multiple hits were observed with $E$ value $<1 \times \mathrm{e}^{-15}$ for large numbers of species, protein sequences of 10-15 representative hematozoa species and 8-10 homologs from other Apicomplexa and/or other eukaryotic organisms were retrieved. Multiple sequence alignments of different proteins were created using Clustal Omega [56]. The alignments were visually inspected for insertions or deletions of fixed lengths which were flanked on both sides by at least $4-5$ conserved amino acids (aa) in the adjacent 40-50 aa and appeared to be exclusive to some or all hematozoa. Query sequences encompassing the indel and its flanking 50-100 aa were collected for all potential CSIs. Afterwards, the query sequences underwent another BLASTp search. The resulting top 500 hits for all queries were examined to determine the presence or absence of CSIs in the homologs from different species and thus the group specificities of the CSIs. Signature files for all CSIs were created using SIG_CREATE and SIG_STYLE programs described in our earlier work [47] that are available on the GLEANS (Gleans.net) server. The CSIs reported here, unless otherwise indicated, are specific for all members of the indicated groups including additional strains for different species (not shown in main Figures but information for them is included in the Supplementary Figures), whose homologs were detected by BLASTp searches.

\subsection{Homology Modelling and Analysis of Protein Structures}

Homology models of two proteins containing the CSIs were created to map the locations of the CSIs within the proteins' structures. A homology model of the $40 \mathrm{~S}$ ribosomal protein $\mathrm{S} 3$ from P. berghei, which contains a one aa insertion specific for all Plasmodium species was constructed based on the solved structure of the homologous protein from Toxoplasma gondii (PDB ID: 5XXU_D) [57]. Similarly, a homology model of the leucine aminopeptidase protein from P. vivax was generated using the available crystal structure from P. falciparum (PDB ID: 4ZX8_A) [58]. Homology modeling was carried out using 
the MODELLER v9.11 program [59] and their stereochemical properties were assessed as described in our earlier work [60].

\section{Results}

\subsection{Phylogenetic Analysis of Hematozoa}

The evolutionary relationships among the genome-sequenced hematozoa species was examined based on two large datasets of concatenated protein sequences each consisting of multiple conserved proteins. The first dataset is composed of 14 proteins which are transcription and translation related, whereas the second dataset contains 10 proteins involved in metabolism and other cellular functions. The second dataset also included the protein adenylosuccinate lyase, which has been previously used in a phylogenetic study on Plasmodium [16,61]. As the two protein datasets utilize proteins involved in different functions, results from them should provide independent assessment of the evolutionary relationships among the hematozoa species.

The phylogenetic trees based on the two protein datasets are shown in Figure 1A,B. Both trees demonstrate highly-supported and consistent interrelationships. In both trees, the Piroplasmida group of species are found to branch closest to the Plasmodium clade, which has also been observed previously $[12,30,31]$. Within the Piroplasmida cluster, species from the genera Babesia and Theileria also form distinct clades, except for the anomalous branching of Babesia microti, which branches deeply and separately from the other Babesia as well as Theileria species. These results are also consistent with earlier studies $[5,8,10,12,33]$. The Plasmodium species form a strongly supported monophyletic clade in both trees and this clade is separated from Piroplasmida by a long branch.

(A)

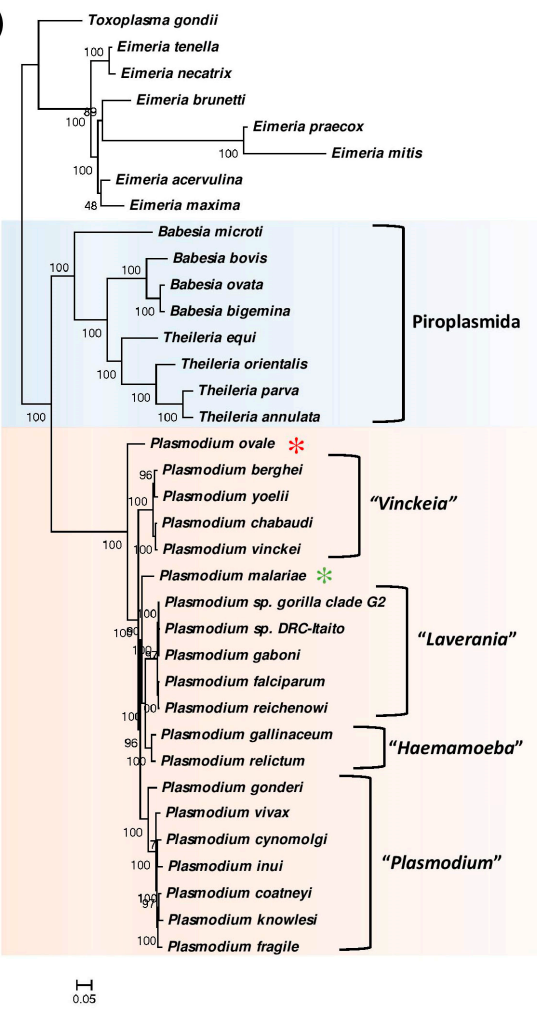

(B)

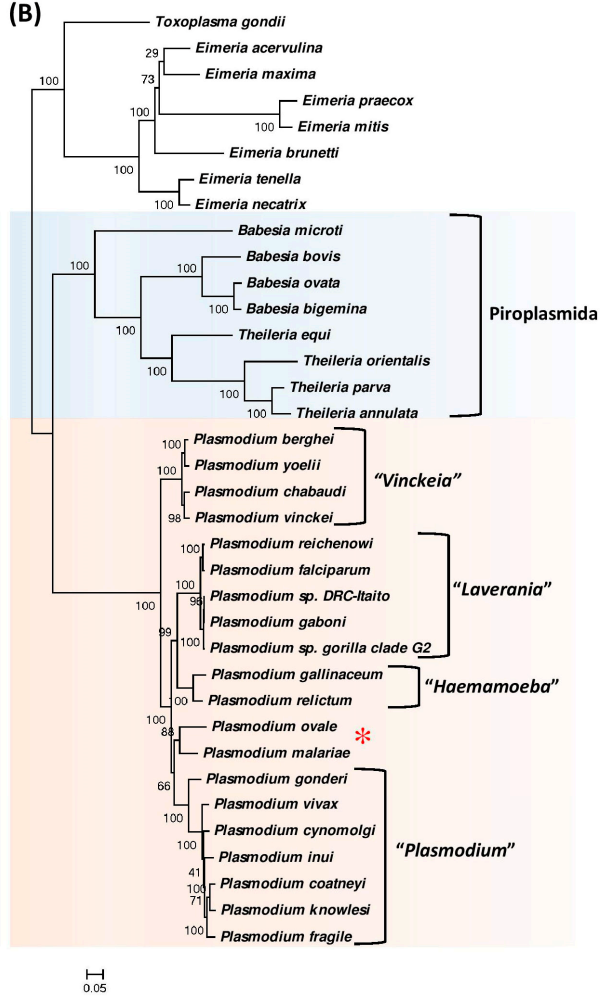

Figure 1. Maximum likelihood trees for the 28 genome sequenced members of the class Hematozoa (A) Tree based on concatenated sequence of 14 transcription and translation related proteins and (B) tree based on concatenated sequence of 10 conserved metabolism-related proteins. The trees were rooted using sequences from other Apicomplexa species. Numbers on the branches indicate bootstrap values for these nodes. Some of the known taxonomic groups are labelled and the Plasmodium species showing anomalous branching are marked with asterisks $\left(^{*}\right)$. 
Although the species within the Plasmodium clade are tightly clustered, in both trees, at least four distinct clades of Plasmodium species are consistently observed. We have labelled these clades based on the names of the subgenera to which most of the species in these groups have been classified $[2,19,23,27,29,34-36]$. These clades include a clade of avian-infecting species labelled as the "Haemamoeba" group and three clades of mammalian-infecting species labelled as the "Vinckeia", "Laverania" and the "Plasmodium" groupings. While all other Plasmodium species group within these four clades, the species P. ovale and P. malariae group separately and their branching positions also differ in the two constructed trees. Whereas in the tree based on transcription-translation related proteins (Figure 1A), P. ovale formed the earliest branching lineage within the genus Plasmodium, in the tree based on metabolism related proteins, P. ovale clustered with P. malariae and this clade branched in the vicinity of the "Vinckeia" subgenus. Although, presently both P. ovale and P. malariae are classified in the subgenus Plasmodium, their taxonomic affiliation or phylogenetic affinity has not been resolved by earlier studies [12,22,23,27-29,62]. Another observation of interest is that in both phylogenetic trees the "Haemamoeba" clade corresponding to the two avian-infecting species does not branch separately from the clades of mammalian-infecting Plasmodium species. Similar branching of the avian infecting Plasmodium species has also been seen in some earlier studies [12,38,39].

\subsection{Identification of Molecular Markers Specific for Hematozoa and its Main Groups}

Based on the phylogenetic trees shown in Figure 1, some inferences regarding the evolutionary relationships amongst the hematozoa species can be drawn. However due to the tight clustering of Plasmodium species in these trees and the short branches which separate the identified clades, it is important to confirm these inferences by other independent approaches. As noted in the introduction, conserved signature indels (CSIs) in protein sequences that are uniquely shared by a given group of organisms provide an important class of molecular markers that have been proven very useful for evolutionary/taxonomic studies [40,41,43]. Therefore, a major focus of the present work was to perform comprehensive genomic analysis on protein sequences from hematozoa species to identify CSIs that are specific for different groups of organisms comprising this class. Our analysis has identified a total of 79 CSIs that are specific for multiple clades and taxonomic groupings of Hematozoa. The characteristics of the identified CSIs and their significance concerning evolutionary relationships amongst the class Hematozoa and the genus Plasmodium are described below.

\subsection{Molecular Markers Specific for the Class Hematozoa}

Our work has identified six CSIs in six different proteins which are specific for the class Hematozoa. An example of one of these CSIs, consisting of a one aa insertion (highlighted) in the cell division cycle protein 48 (Cdc48), is presented in Figure 2. The one aa CSI in this protein is present in a conserved region and it is commonly shared by all homologs from the class Hematozoa for which sequence information is available, but it is not present in any other Apicomplexa species or the homologs of other eukaryotic organisms present in top BLASTp searches. The Cdc 48 protein is an ubiquitin-dependent molecular chaperone, which help mediate a variety of degradative and regulatory processes in order to maintain cellular homoeostasis [63]. Similar to this CSI, five other CSIs which are also specific for the class Hematozoa were identified the present work. These CSIs are found in the proteins 20S proteasome $\beta 4$ subunit, a putative 30S ribosomal protein S9, a putative 40S ribosomal protein S12, Golgi reassembly-stacking protein 1 and pyruvate kinase 2A. Some characteristics of these CSIs are summarized in Table 1 and detailed sequence information for them is provided in Figures S1-S6. Due to the specificities of these CSIs for the class Hematozoa, the genetic changes associated with these CSIs likely occurred in a common ancestor of this class. 

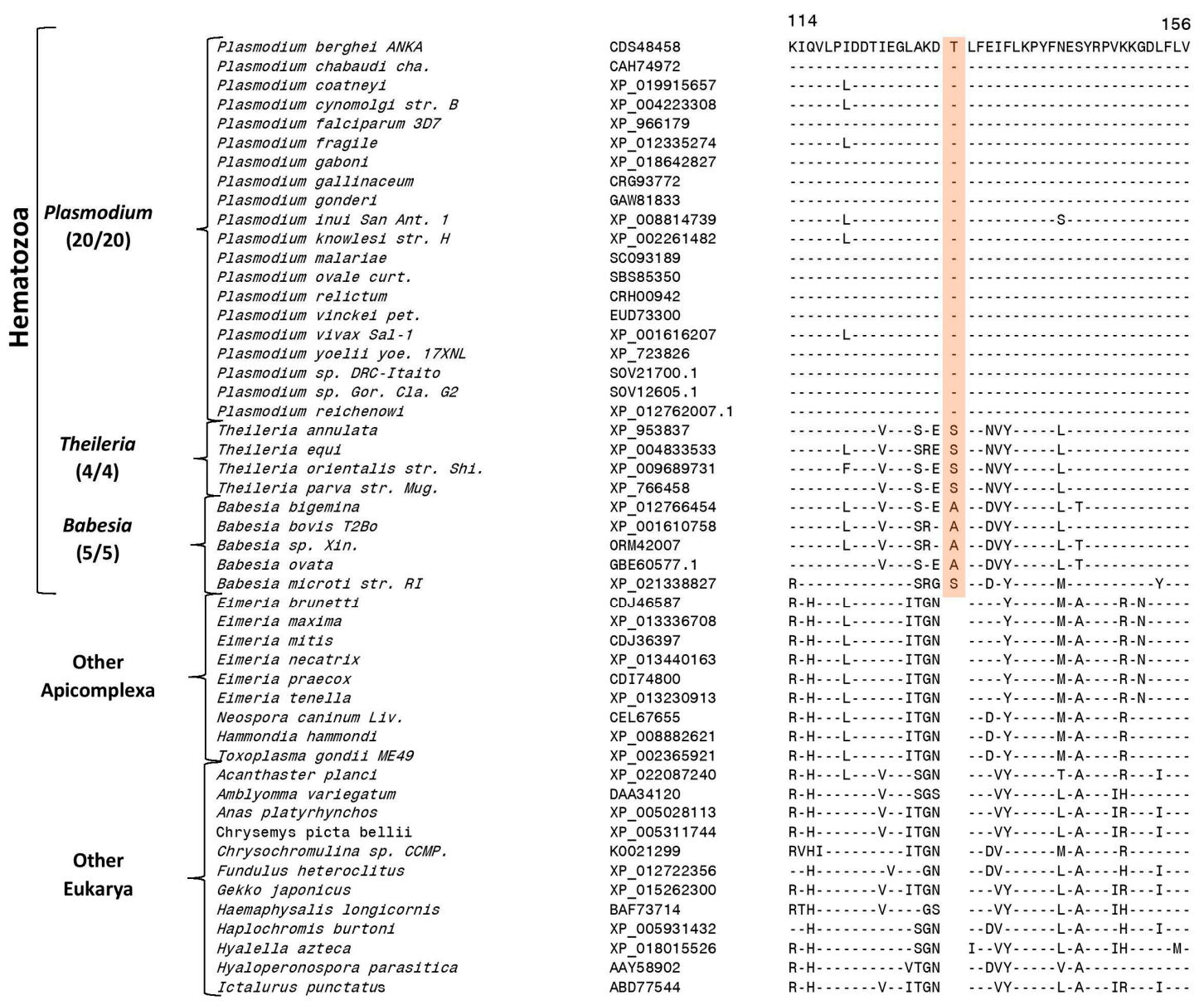

Figure 2. Partial sequence alignment of the cell division cycle protein Cdc48 showing a 1-aa insertion in a conserved region (boxed) which is exclusively found in all available homologs from members of the class Hematozoa. The dashes (-) in the alignment indicate identity with the amino acid residues shown in the top sequence. Accession numbers for each sequence are indicated in the second column. Information for five additional CSIs that are also specific for the class Hematozoa are shown in Figures S2-S6 and information for them is summarized in Table 1. The numbers with the group names in all figures indicate the presence/absence of the CSIs in different species which are shown in Figure 1. In cases, where sequences for additional strains from these groups were detected by BLASTp searches, they also contained the indicated CSIs (see Supplementary Figures). 
Table 1. Summary of CSIs specific for the class Hematozoa and order Piroplasmida.

\begin{tabular}{|c|c|c|c|c|c|}
\hline Protein Name & Accession Number & Figure Number & Indel Size & Indel Position & Specificity \\
\hline Cell division cycle protein 48 homologue & CDS48458 & $2, \mathrm{~S} 1$ & 1 aa ins & $106-156$ & \multirow{6}{*}{$\begin{array}{c}\text { Class } \\
\text { Hematozoa }\end{array}$} \\
\hline $30 \mathrm{~S}$ ribosomal protein $\mathrm{S} 9$, putative & CDU21364 & $\mathrm{S} 2$ & 1 aa ins & $449-503$ & \\
\hline $40 S$ ribosomal protein $S 12$, putative & XP_677491 & S3 & 1 aa ins & $62-109$ & \\
\hline $20 \mathrm{~S}$ proteasome $\beta 4$ subunit & CDS50559 & S4 & 1 aa ins & $04-59$ & \\
\hline Golgi reassembly-stacking protein 1 & XP_012769739 & S5 & 1 aa del & $90-136$ & \\
\hline Pyruvate kinase 2 & XP_680419 & S6 & 1 aa ins & $461-515$ & \\
\hline Succinyl-CoA synthetase $\beta$ chain & XP_004833199 & $3(\mathrm{~A}), \mathrm{S} 7$ & 1 aa del & $273-315$ & \multirow{2}{*}{$\begin{array}{c}\text { Order } \\
\text { Piroplasmida }\end{array}$} \\
\hline Hypothetical protein, conserved & XP_954617 & S8 & 1 aa ins & 1856-1907 & \\
\hline Dihydrolipoamide dehydrogenase & XP_763245 & 3 (B), S9 & 1 aa del & $415-465$ & \multirow{5}{*}{$\begin{array}{c}\text { Order } \\
\text { Piroplasmida, } \\
\text { except } B . \\
\text { microti }\end{array}$} \\
\hline Conserved hypothetical protein & XP_004832720 & S10 & 1 aa del & $19-74$ & \\
\hline Conserved hypothetical protein & XP_004832649 & S11 & 2 aa del & $334-388$ & \\
\hline Intron-binding aquarius $\beta$ like & XP_012767741 & S12 & 2 aa del & $1280-1322$ & \\
\hline Intron-binding aquarius $\beta$ like & XP_012767742 & S13 & $5 / 8$ aa del & $1303-1346$ & \\
\hline Cysteinyl-tRNA synthetase & XP_001608890 & S14 & 2 aa ins & $261-316$ & $\begin{array}{c}\text { Genus Babesia } \\
\text { (except } B \text {. } \\
\text { microti) }\end{array}$ \\
\hline Cysteinyl-tRNA synthetase & XP_001608890 & S14 & 1 aa ins & $261-316$ & \multirow{2}{*}{ Genus Theileria } \\
\hline Eukaryotic translation initiation factor $4 a$ & XP_764692 & S15 & 1 aa del & $225-279$ & \\
\hline
\end{tabular}

\subsection{Molecular Signatures Specific for the Order Piroplasmida}

Our analysis has also identified two CSIs which are exclusively found in all available Piroplasmida homologs. An example of one of the CSIs specific for the order Piroplasmida is shown in Figure 3A. In this case, a one aa deletion in the protein succinyl-CoA synthetase $\beta$ chain is uniquely present in the homologs of all Piroplasmida species for which sequence information is available, but this deletion is not found in the protein homologs from other Apicomplexa and Eukarya. Sequence information for this CSI and one other CSI showing similar specificity is provided in Figures S7 and S8 and their main characteristics are summarized in Table 1. The genetic changes leading to these CSIs have likely occurred in a common ancestor of the order Piroplasmida. We have also identified five other CSIs that are commonly shared by all Piroplasmida species except B. microti, which shows deeper branching in the trees in comparison to the other species from this order (Figure 1). One example of a CSI showing this pattern is shown in Figure 3B. In this case, a one aa deletion in a conserved region of the protein dihydrolipoamide dehydrogenase is commonly and uniquely shared by all other Babesia and Theileria species, except B. microti. More detailed sequence information for this CSI and the other four CSIs showing similar species distribution pattern is presented in Figures S9-S13 and some of their characteristics are summarized in Table 1. The genetic changes responsible for these CSIs are postulated to have occurred in a common ancestor of the other Piroplasmida, after the branching of B. microti.

Our analysis has also identified three CSIs which are exclusively found in all sequenced species of either Theileria or Babesia (excluding B. microti) genera. Sequence information for these CSIs is presented in Figures S14 and S15, their main characteristics are also summarized in Table 1. These CSIs serve to distinguish members of the genera Babesia or Theileria from other Apicomplexa and they also provide strong evidence that $B$. microti is genetically distinct from other members of the genus Babesia and makes a case for its placement into a separate genus. 


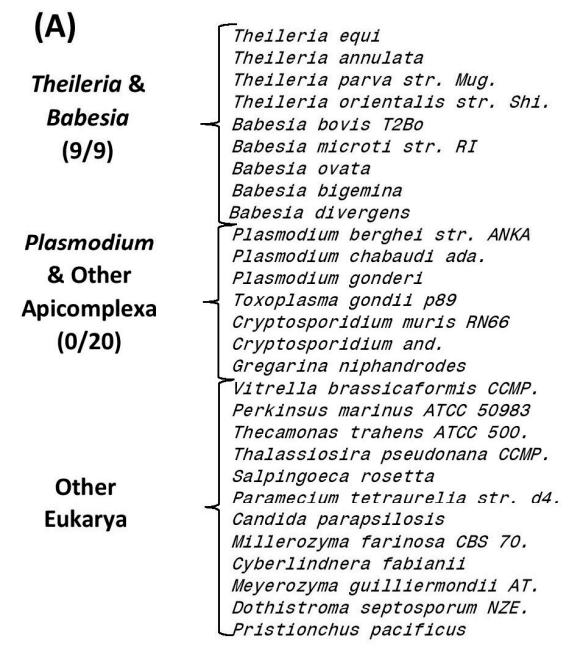

(B)

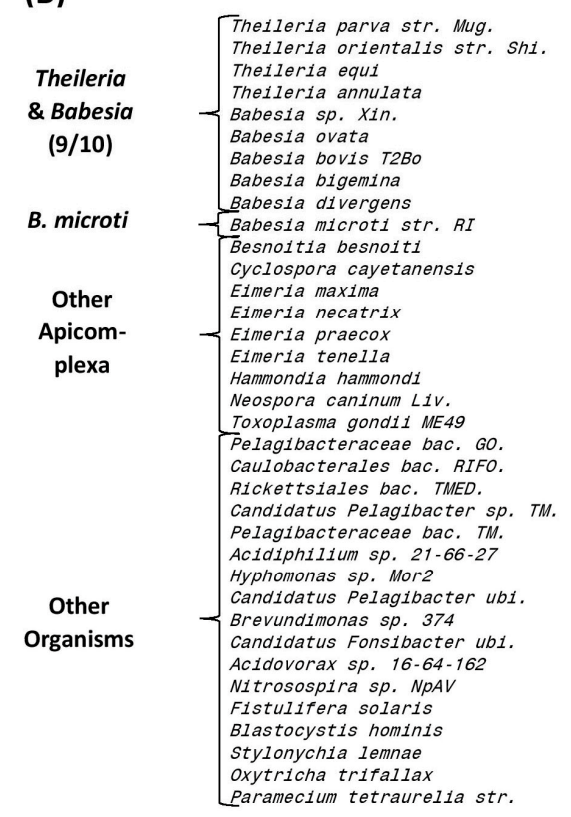

XP_004833199
XP_954105
XP_766198
XP_009689511
XP_001610970
XP_012649299
GBE600307.1
XP_012766706
LK934712.1
XP_677913
SCM07089
GAW82919
672267444
XP_002141262
1098427798
749158289
CEL94328
XP_002783211
XP_01375650
XP_002286405
XP_004996763
XP_001440162
CCE42831
CCE82955
CDR37591
EDK39982
EME41937
PDM72854

273

315

DDNAKYRQTEIFEKSPAN LTHEEAEADKVGLNYISLDGNVACI

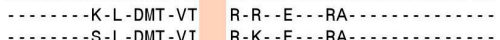

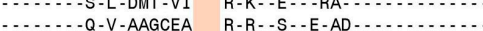
-..-DF-.K-..-SV-PA ENEL-R-NE-.......-G-I -.. ...DF-D-Y-KNA-MP VNPA-LA-KNA-V -..-DF--KD-ASVAPP ENEL-R-VEA-............ -.-DF--KD-ASVAPP ENEL--R-LEA-....P-G-....

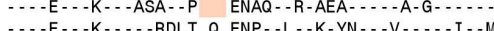

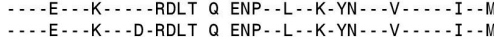

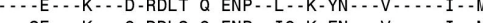

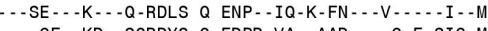
-.-GF--KD--SQRDYS Q EDPR-VA--AAD-..-G-E-SIG-M -S-.-K-L-DIEDVE Q KDQI -V-.A-Y....-R-N-..-G-S -..-K-L-DIEDVE Q KDQI -V - -A-Y....-R-N-.-G...FF.-K-L-AQEDTS V RCPR-LA-EAEHM-.V-M-...-G-L -S -...-Q-L-SMEDES L MDPR - -A-H-A-...-G-..-IG-L -.-QF--KD--SQRDIT Q EDPR-VA-S-WD....-G-...IG-II - AF - - AMRDTT E EDPRDV S-HD -

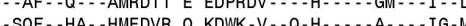
-AF - Q Q-V-SWRDPT Q EDPQ -...S-Y...F-K -SF-.E-V-SWRDPT Q EDPQ - A-Y -F-K - I N-

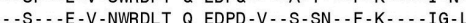
-SF.-K-V-SWRDPT Q EDP.-V..-AEY...F-K....I-N ....EF-.K -..SWRDPS Q EDAD-VK-GE.....F-K...DIG-L

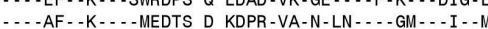

415 457

\begin{tabular}{|c|c|c|}
\hline & SRAKIYNESDGFIKLLSTEEN & KLLGAWMIGP \\
\hline XP_009691656 & $-A-\ldots . .-G-T-. V-.-A D-A-$ & R...-LV - . A - . - V-Q \\
\hline XP_-004832497 & $Y-G-\ldots .-G-. .-V-I-A D K--$ & - I - . - IV- - DA - -L-AQ \\
\hline $\mathrm{XP}_{-}^{-} 954919$ & - TE- -V - AN-Q- & ........... \\
\hline ORM̄40305 & $\cdots$ - R-AG-T- - -V-I - ADKD - & $-I--G-I V-\ldots A--L-C$ \\
\hline GBE62323 & -A....-RVAG-T $\ldots$ - V-I -ADND - & $-I--G-I V-\ldots A--L-G Q$ \\
\hline XP_001610643 & -A- - - R-AGDV - - - - I - ADKD - & $-I--G-I V--A--L-G Q$ \\
\hline$X P_{-}-012769794$ & - A - . - SRVAG - T - . - V - I - ADND - & - I - - G-I - - -QA - -L-GQ \\
\hline LK9 34716.1 & - - SR - AE - TE - -V - I - ADKD - & -I- - G-IV-.-A- - LVGQ \\
\hline & $\cdots$ LA- -TT - -V -V - - D - -S & $G-I-\ldots I F-T Q A--L-A-$ \\
\hline $\mathrm{PF} \overline{\mathrm{H}} 35994$ & -A- . - RANDVVQ - -V - I -TDK-T & \\
\hline XP_022589645 & $-V-\ldots$ RATGD $\ldots$ - IV-V - ACK - T & D …-V-IL-QGAG-L-AE \\
\hline XP-013332622 & -A $\ldots$ RATGD $\ldots$ - LV - V-TCK - T & D - I - - V-IL- -NAG-L- \\
\hline XP & -A.... RATGD - . -LV-V-TCK-T & \\
\hline CDĪ86766 & -A $\cdots \ldots$ RATGD $\cdots$ - IV-V-TCKDT & D - I - -V-IL- -NAG-L-AE \\
\hline XP_013229686 & -A $\ldots$ RATGD $\cdots$ - LV-V-TCK-T & $-I--V-I L--N A G-L-A E$ \\
\hline XP_-008886018 & -A-... RANDVAT - -V-V-AHKDS & D $\ldots$. IIM- - EAG -L-GQ \\
\hline CEL-68674 & -A- - RANDVAT - -V-V-AHK-S & D - I - . IIM- - EAG -L-GQ \\
\hline XP_002367828 & -A- . - RANDVAT - -V-V-AHKDS & D $\ldots$. I - IM- - EAG-L \\
\hline & -AID - PE - - V - I - ADQST & D $-V-V H I \cdots A G \cdots A E$ \\
\hline OGN49878 & $-T-N H-T-V-V$-ADAAT & D $-V-$-VHIM - -QAG $\cdots-E$ \\
\hline OUW24307 & V VN- - TE- - V-I - ANRDT & D $-V--V H I-\ldots$ CGD - -A \\
\hline OUW48613 & $-L \cdots+V N-\ldots E-V-I-A D A R T$ & D -V - -VHI - . - CGD - -AE \\
\hline OUX32806 & -I -1 - AI - - PE - -V - I -AD-KT & D -I - -VH-...-G-I-GE \\
\hline oYv666 & $\ldots$ - G- RAMG - T $\ldots$ - V $\ldots$ DKTT & D $\ldots$ - HI $\ldots$ DAGTI -AE \\
\hline WP_07C & -RTNH - TA- - V - I - AE - GT & $D-I \cdots-H-V G-G-$ \\
\hline WP_075534421 & $\cdots$ AI - -AE- -V-I - AD - KT & D -V - - VHL ... - AG-L-AE \\
\hline WP_091750748 & $-\mathrm{T} \cdot \ldots-\mathrm{NH}-\mathrm{T} \cdot-\mathrm{V}-\mathrm{V}-\mathrm{ADAAT}$ & D -V - -VHIM- - QAG - ... \\
\hline WP_-0993 & -A- -A- - VND -G- - V-I - D - KT & RV - - VH - . - D - G \\
\hline OYZ̄ 44529 & TT - MV - F - ADAVT & $E I-. V H-V-. Q-$. \\
\hline WP_041514166 & $\cdots-$ G- -RALG-TG- -V-V-ADVDT & D RI - V VH.... Y $\ldots .$. \\
\hline GĀ̄ 16732 & -S....-RANATT $\cdots$ - V-V-ADA-T & D RI - - CHIM- -NAG -. \\
\hline ABU54774 & LQ - . - - RA-DQP - - L- - V-ADKKT & $N-I--V H-C-L N-.-L$ \\
\hline CDW75606 & RTN-D-E-LV-V-ADKDT & D - I - - VHI - - -NAG \\
\hline EJY727 & -Q_...-RAN-D -..LV-I - TDQQS & G $-I \ldots H I \ldots N A G$ \\
\hline XP 001 & 一 AND - IE $\ldots$ - - V-TDKKT & \\
\hline
\end{tabular}

Figure 3. Sequence alignments showing conserved signature indels specific for members of the order Piroplasmida. (A) Partial sequence alignment of the protein succinyl-CoA synthetase $\beta$ chain showing a 1-aa deletion that is uniquely shared by all available homologs from the order Piroplasmida. (B) Partial sequence alignment of the protein dihydrolipoamide dehydrogenase showing a 1-aa deletion in a conserved region that is present in all Piroplasmida homologs except Babesia microti. Sequence alignments for multiple other CSIs exhibiting similar specificities is provided in Supplementary Figures S7-S13 and their main characteristics are summarized in Table 1.

\subsection{Molecular Signatures Specific for the Genus Plasmodium and Its Subgenera}

The present work has also identified large numbers of CSIs which are specifically shared by either all or specific groups of Plasmodium species providing novel means for their identification and understanding their interrelationships. Of these CSIs, 23 CSIs present in different proteins are exclusively shared by the homologs of all genome sequenced Plasmodium species, but not found in any other Apicomplexa or metazoan species. Figure 4A shows one example of a CSI, consisting of a one aa insertion in a highly conserved region of the $40 \mathrm{~S}$ ribosomal protein $\mathrm{S} 3$, which is specifically shared by all available Plasmodium homologs, but not found in any other eukaryotic organism. 


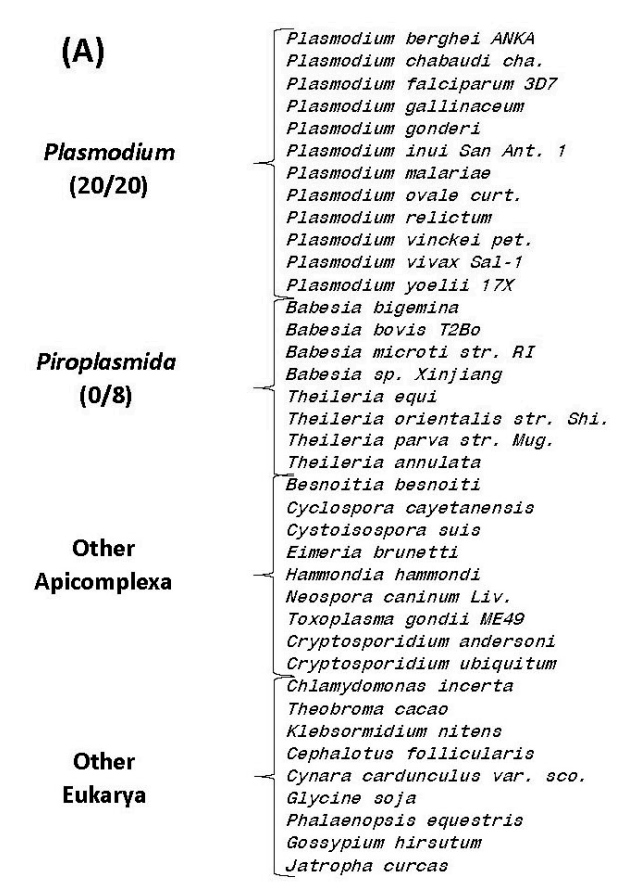

CDS50468
XP_016654586
XP_001348801
CRG94856
GAW82426
XP_008813962
SBT0043
SBS86392
CRH01265
EUD70471
XP_001615729
ETB63049
XP_012767161
XP_001609690
XP_012648260
ORM41413
XP_00431148
XP_009692420
XP_764056
XP_953083
PFH38580
XP_022592372
PHJ24329
CDJ53616
XP_008885620
XP_003883474
XP_018636818
OII 75791
OII74098
ABA01099
EOY30019
GA080594
GAV81193
KVH95611
KHN24447
XP_020578106
XP_016719678
XP_012077022

50 93

IRATRTREYLGDKGRR IRELTSLVQKR F FNKLTNSYELFAERVE
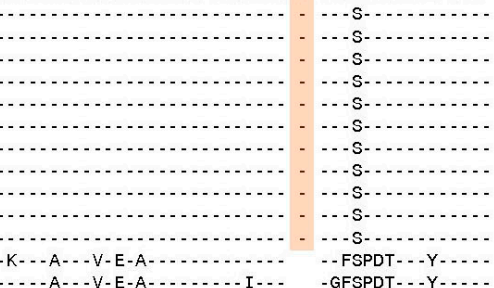

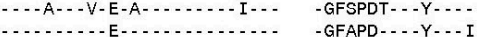

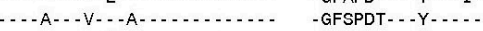

-GFSSD-...Y-...

E-A-

E-A

GFSSD - ...Y....

GFSSD - . -Y. - .

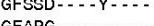

GFAPG-

GFPPD-..-

GFAPD

-AFAPD-.

-GFAPD-.

-GFAPD-

GFAPD-......
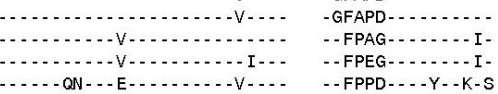

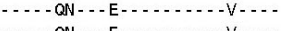

-
-

-KFAE -...YY-K-N

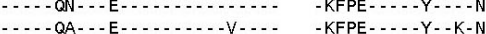

-

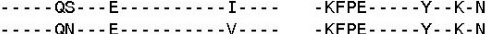

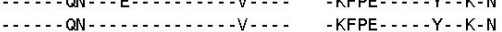

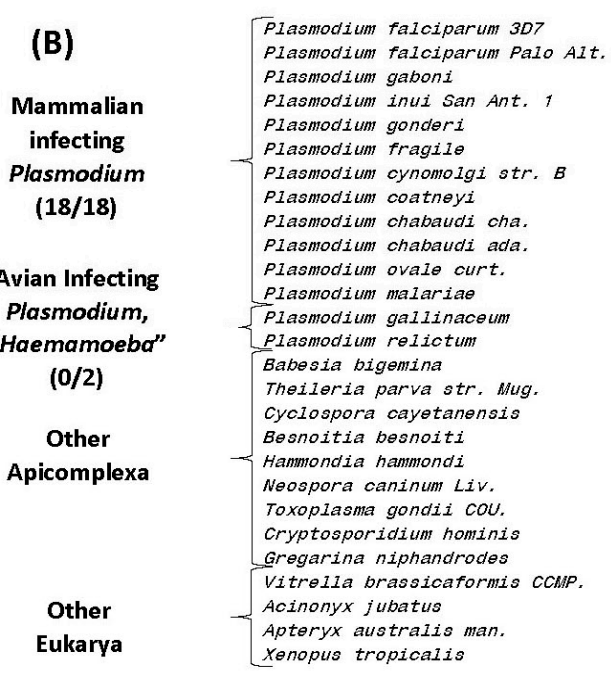

142

185

XP_024329092 DIVKYIEKIIENKYAYVS E EGSVYFDIDEFKKSEKHFYARMEPL ETW56568

XP_O18641535 XP_o08815940 GAW 80575

XP_012337164

XP_-004222036

SCM 24562

SCM24562

SCN61934

SBS82865

SBS84520

CRG94210

CRG99705

$X P \_012767680$
$X P-763084$

XP_-022586743

$\mathrm{PFH} 37612$

XP_o08888417

CEL70364

PIL96768

OLQ17505

XP 011131114

CEN20223

XP_014936514

XP_013813315

OCA14793

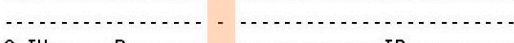

Q- IH

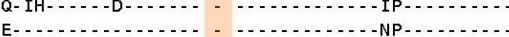

Q-IQ- Q Q . .

Q

Q- IO-

E-I-F-C

E-I-F

E-

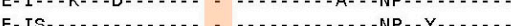

E-IS- .

- I I -

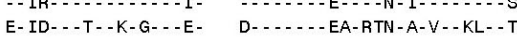

E- -DF-K- - LD-G- - D D - D - - - EA-RS-G-V-V-KL- - T

EVLRL- -E-EA-GF--E- Q Q-...-TQR-S- -A-T-T-G-..-G

EVLSL- QR - EK-G- - -E- Q-...--TQS-RN-D- - I-G-..-S

EV-AL- -R-VK-G-G-E- Q-...--TQT-R-N-.-V-G-..-T

EVLSL- -R-VK-G-G-E- Q-...-TQT-R-TD-. I-G-..-S

EV-AL- -R-VK-G-G-E- Q-...-TQT-R-N-.-V-G-..-T

E- IDF-ST - SRGF- - E- - - - - - VES-RAD- - V-G- - -K

E-Q-F-L. - -D-G- - E- G- ...-TTA-RN-..-V-G- ..-W

EV-GF-Q. - -D- ...-E- S- . . - VAA- -S-GQ-V-G- .. -W

E. - NFVQ- -VD-G-G- . - N - . . . - TVK-AS- -R-S-GKLV -E

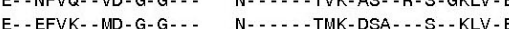

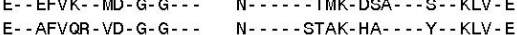

Figure 4. Sequence alignments of conserved signature indels specific for the genus Plasmodium and one that is exclusively found in the mammalian-infecting species. (A) Partial sequence alignment of the 40 S ribosomal protein S3 showing a 1-aa insertion in a conserved region that is uniquely found in all available Plasmodium homologs. Sequence information for 22 other CSI in other proteins that are also specific for the genus Plasmodium is shown in Figures S17-S38 and their summary is provided in Table 2. (B) Partial sequence alignment of the protein cysteine-tRNA ligase showing a 1-aa insertion that is uniquely shared by all other Plasmodium homologs, but lacking in the avian-infecting species (subgenus Haemamoeba) as well as other eukaryotic organisms. 
Table 2. Summary of CSIs specific for the Genus Plasmodium and some of its clades/groups.

\begin{tabular}{|c|c|c|c|c|c|}
\hline Protein Name & Accession Number & Figure Number & Indel Size & Indel Position & Specificity \\
\hline $40 \mathrm{~S}$ ribosomal protein $\mathrm{S} 3$ & CDS50468 & $4(\mathrm{~A}), \mathrm{S} 16$ & 1 aa ins & $50-103$ & \multirow{23}{*}{$\begin{array}{c}\text { Genus } \\
\text { Plasmodium }\end{array}$} \\
\hline $26 \mathrm{~S}$ proteasome regulatory subunit RPN2 & CDS50476 & S17 & 2 aa ins & $777-826$ & \\
\hline $26 \mathrm{~S}$ proteasome regulatory subunit 4 & XP_673015 & S18 & 1 aa del & $64-116$ & \\
\hline $40 \mathrm{~S}$ ribosomal protein $\mathrm{S} 25$, putative & XP_001348379 & S19 & 1 aa del & $35-79$ & \\
\hline $50 \mathrm{~S}$ ribosomal protein $\mathrm{L} 1$, mitochondrial, putative * & XP_674529 & $\mathrm{S} 20$ & 1 aa del & $126-167$ & \\
\hline $60 \mathrm{~S}$ ribosomal protein $\mathrm{L} 35$, putative & XP_001347931 & S21 & 1 aa del & $25-64$ & \\
\hline Asparagine-tRNA ligase, putative* & XP_680201 & S22 & 1 aa del & $436-487$ & \\
\hline ATP-dependent RNA helicase DBP10 & CDS45652 & S23 & 1 aa ins & $385-438$ & \\
\hline Alternative splicing regulator, putative & XP_001349840 & S24 & 1 aa del & $296-338$ & \\
\hline Adenosinetriphosphatase protein & XP_677822 & S25 & 1 aa del & $387-437$ & \\
\hline DNA2/NAM7 helicase & CDS49097 & S26 & $3 / 4$ aa ins & 950-1013 & \\
\hline Elongation factor Tu family & CDS46726 & S27 & 1 aa ins & $335-380$ & \\
\hline Multidrug resistance protein 2 & CDS50130 & S28 & 1 aa del & $438-488$ & \\
\hline Pre-mRNA-processing-splicing factor 8 & XP_022714222 & S29 & 2 aa ins & $1174-1230$ & \\
\hline Pyruvate dehydrogenase E1 component, $\alpha$ subunit & CDS46715 & S30 & 1 aa ins & $218-272$ & \\
\hline Pyruvate dehydrogenase E1 component, $\alpha$ subunit & CDS46715 & S31 & 1 aa ins & $406-451$ & \\
\hline Ras-related protein Rab-5A & XP_677676 & S32 & 2 aa del & 103-162 & \\
\hline Ribosomal protein L27a, putative & XP_677367 & S33 & 1 aa ins & $97-142$ & \\
\hline RuvB-like helicase isoform 1 & XP_673190 & S34 & 1 aa ins & $121-178$ & \\
\hline RuvB-like helicase isoform 1 & XP_673190 & S35 & 1 aa del & 96-144 & \\
\hline RuvB-like helicase isoform 2 & CDS46888 & S36 & 1 aa ins & $136-194$ & \\
\hline Splicing factor 3A subunit 2 & XP_676664 & S37 & 1 aa del & $111-157$ & \\
\hline Translation initiation factor SUI1 & SCM24886 & S38 & 1 aa del & $181-221$ & \\
\hline Cysteine-tRNA ligase protein & XP_024329092 & 4 (B), S39 & 1 aa ins & $135-185$ & $\begin{array}{l}\text { Mammalian } \\
\text { infecting clades }\end{array}$ \\
\hline Protein phosphatase & CRH00140 & $5(\mathrm{~A}), \mathrm{S} 40$ & 1 aa ins & $146-197$ & Avian clade, \\
\hline Conserved Plasmodium protein & CRG96454 & S41 & 1 aa ins & 5-58 & "Haemamoeba" \\
\hline
\end{tabular}

* CSI region is only conserved within Plasmodium and various bacteria.

Sequence information for the 22 other CSIs present in other important proteins that are also distinctive characteristics of the genus Plasmodium is presented in Supplementary Figures S16-S38 and some of their characteristics are summarized in Table 2. Due to the exclusive presence of these CSIs in the homologs from all sequenced Plasmodium species, the genetic changes leading to these CSIs have likely occurred in a common ancestor of the genus Plasmodium, providing important molecular characteristics distinguishing this group of organisms from all others.

Another interesting CSI identified by our analysis found in a highly conserved region of the protein cysteine-tRNA ligase is uniquely shared by all mammalian-infecting Plasmodium species, but it is not found in the members of the subgenus "Haemamoeba" consisting of the two avian infecting species viz. P. gallinaceum and P. relictum (Figure 4B) (a more detailed alignment can be found in Figure S39).

The absence of this CSI in all other Apicomplexa as well as eukaryotic organisms indicates that this CSI represents an insert and the genetic change responsible for this CSI occurred in a common ancestor of the mammalian-infecting Plasmodium species after this group of organisms diverged from the avian-infecting Plasmodium species. Thus, this CSI supports the inference that the avian-infecting species are ancestral to the mammalian-infecting Plasmodium species [39,64].

We have also identified multiple CSIs that are specific for the four main groupings or subgenera of Plasmodium species. Two of these CSIs are specific for the subgenus "Haemamoeba" comprised of $P$. relictum and $P$. gallinaceum species.

Sequence information for one of these CSIs consisting of a one aa insert in a protein phosphatase that is exclusively found in these two avian-infecting Plasmodium species, but not in any other Plasmodium or Apicomplexa species is presented in Figure 5A. Sequence information for the other CSI specific for the "Haemamoeba" clade is provided in Supplementary Figure S41. 
(A)

$\begin{array}{cl}\text { "Haemamoeba" } & \left\{\begin{array}{l}\text { Plasmodium relictum } \\ \text { Plasmodium gallinaceum }\end{array}\right. \\ \text { Other } & \begin{array}{l}\text { Plasmodium berghei ANKA } \\ \text { Plasmodium cynomolgi str. B } \\ \text { Plasmodium falciparum } 3 D 7 \\ \text { Plasmodium fragile } \\ \text { Plasmodium gaboni }\end{array} \\ \text { (0/18) } & \text { Plasmodium malariae } \\ \text { Plasmodium ovale curt. } \\ \text { Plasmodium vinckei pet. } \\ \text { Plasmodium vivax North Kor. } \\ \text { Plasmodium yoelii yoe. }\end{array}$

(B)

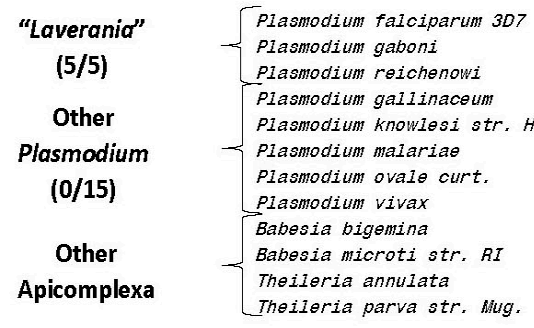

(C)

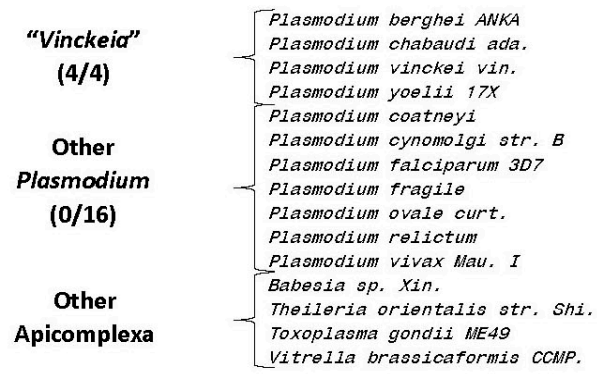

(D)

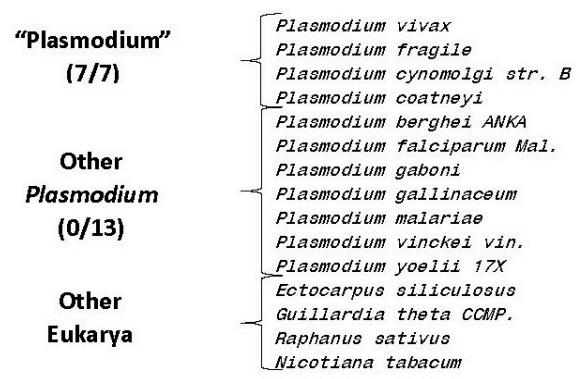

CRH00140
CRG96319
XP_022712909
XP_004222493
XP_001347952
XP_012333086
SoV15735
SBS85607
SBS83709
EUD72878
KMZ99533
EAA21549

XP_001347362
SOV14644
XP_012763117
CRG94136
XP_002258710
SBT00797
SBS82818
XP_001614386
XP_012768468
XP_012650506
XP_955469
XP_762911

158

KKSNIDNQL ILGYKD S DPFDLDNKEMMEKLKSLAWNTDDI -EA-V-.....-R- K - - Y- - KKE-L-T- - -N-T-.... NNI- - T- YNL - - THI N-YEI-S- IRDKE-AT-N-DS-NL RDA- - V- - VL- - HEE - - Y-V-S-TLENE----N-D- - KL - -N- - F-HI- V-HQE N-Y- INED-IDD- - - MS - . - NL -DASVV-EV- - - HQE - - YEV-A-TLENE-.- -N-D- - KL - - N- - FHH I - . - HQE N-Y- INED-LND- - N-MS- ... - NL

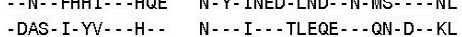

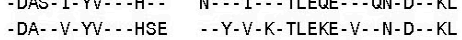

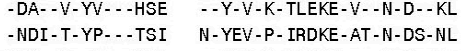
RDA- -V- - VL--HQE - - YEV-S-TLENE--.-N-D-- QL NNI- - T-YNL- - THI N-YEV-S- IRDKE-AT-N-DS-NL

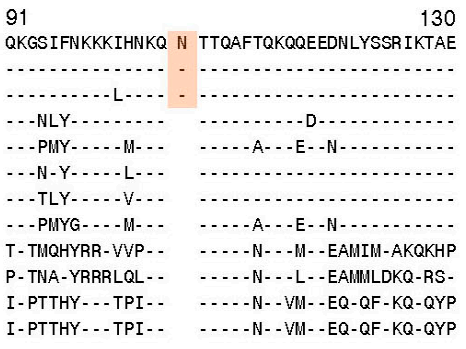

53

92

\begin{tabular}{|c|c|c|}
\hline XP_022714285 & AKELQGYAEELIYLA K & KKNNSENSLLVESILRTAQGRRKL \\
\hline scī07120 & & \\
\hline XP_008623859 & - & -.... \\
\hline ETB59742 & \multirow{2}{*}{ - } & 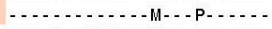 \\
\hline XP_019917193 & & 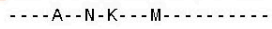 \\
\hline XP_-004224350 & (- & 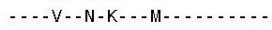 \\
\hline XP_-001348463 & - n & $--D-V--N-K--M-C-R-R-$ \\
\hline XP_012338668 & \multirow{2}{*}{ n } & 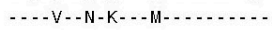 \\
\hline SBS̄87294 & & - - DSE--K-K- - M- \\
\hline CRHO3842 & - & 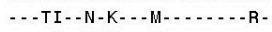 \\
\hline KMZ90929 & - & $-A_{-}-N-K--M-\ldots$ \\
\hline ORM39458 & - - - Q - - WVFH & - - DTP-SD-V - .-M - . PEA-HE- \\
\hline XP_009689506 & \multirow{2}{*}{ 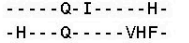 } & -RDTH-SD - I - - VI - - PES-SL- \\
\hline XP_002371147 & & -Q-TP-S- - I - - MIF- PAA- . - \\
\hline \multirow[t]{2}{*}{ CEM12687 } & - - - Q- - IVF- . & - -DTP-SD-K- - -M- - - PAA- - I- \\
\hline & 362 & 401 \\
\hline 56068989 & \multicolumn{2}{|c|}{ PNKFIHLTYKG AQTGA SQNEKKKIALIGKGITFDSGGYNL } \\
\hline XP_012337457 & - . - K & NK- \\
\hline XP_004224071 & $--K-D$ & $\mathrm{~N}-\mathrm{-KR}-$ \\
\hline XP_019916508 & -...-HK-D & $K--K V-\ldots . . .$. \\
\hline XP_678034 & - - R - - - - - & KGDI $\ldots . . .-. .$. \\
\hline ETW'46651 & 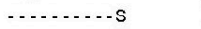 & KGDV $\ldots$ \\
\hline Sov19335 & 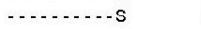 & KGDV $---V--V-\ldots$ \\
\hline CRG94657 & 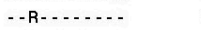 & NGEI $-[-V--V-\ldots \ldots$ \\
\hline SBS88844 & & KGDI $\ldots \ldots$ V \\
\hline XP_008622014 & $-H R-$ & KGDI $\ldots \ldots-V_{-\ldots}$ \\
\hline ЕTВ556248 & - R-A..... & KGDI $-\ldots$ \\
\hline CBJ32669 & $-P-\ldots+P^{\prime}$ & -GTP-- V \\
\hline XP_005835498 & $-P-\cdots--V--P$ & KGEV - \\
\hline XP_018464138 & $-P F-\ldots V-R P$ & 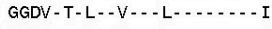 \\
\hline AGW47884 & $-P H-\cdots-C-P$ & GGEI - - L- - V-- -L- - - - - - I I \\
\hline
\end{tabular}

Figure 5. Examples of CSIs that are specific for different groups/subgenera of Plasmodium species (A) Partial sequence alignment of a protein phosphatase showing a 1-aa insertion that is specific for the subgenus Haemamoeba. (B) Partial sequence alignment of the eukaryotic translation initiation factor 3 (subunit D) showing a 1-aa insertion that is specific for members of the subgenus Laverania. (C) Partial sequence alignment of the mitochondrial ribosomal protein L17-2 showing a 1-aa insertion that is specific for the subgenus Vinckeia. (D) Partial sequence alignment of the protein leucine aminopeptidase containing a 5-aa insertion exclusively found in members of the subgenus Plasmodium except P. ovale and $P$. malariae. Sequence alignments for multiple other CSIs that are also specific for these subgenera are provided in Figures S41, S60-S66 and information for them is summarized in Table 3. 
Table 3. Summary of CSIs specific for mammalian-infecting Plasmodium clades.

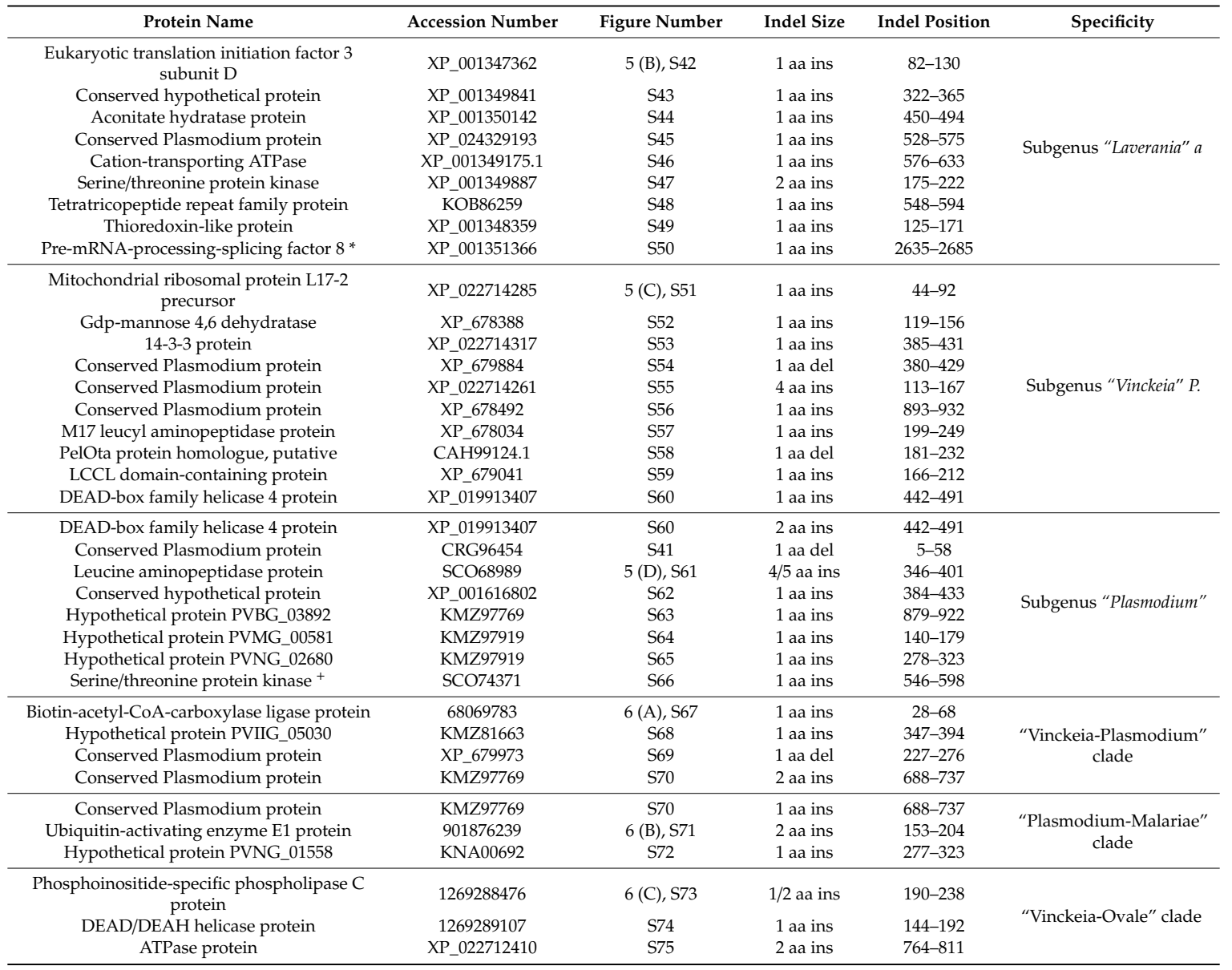

${ }^{*}$ CSI is specific for a subclade of Laverania consisting of P. falciparum and P. reichenowi; ${ }^{+} \mathrm{CSI}$ is also shared by some Laverania species.

Nine other CSIs identified in this work are exclusively shared by members of the subgenus "Laverania". This group is made up of the following genome sequenced species: P. falciparum, P. reichenowi, P. gaboni and two unnamed species P. sp. gorilla clade G2 and P. sp. DRC-Italio. One example of a CSI consisting of a one aa insertion present in the eukaryotic translation initiation factor 3 subunit D, which is specific for this group is shown in Figure 5B. More detailed sequence information for this CSI as well as the remaining eight CSIs that are specific for the "Laverania" clade is provided in Figures S42-S50 and some characteristics of these CSIs are summarized in Table 3. It is of interest to note that for one of the CSIs specific for this group, found in the protein pre-mRNA processing splicing factor 8 (Figure S50), is uniquely shared by all other members of this group except $P$. gaboni, $P$. sp. gorilla clade G2 and P. sp. DRC-Italio. This CSI suggests that within the "Laverania" group, P. gaboni P. sp. gorilla clade G2 and P. sp. DRC-Italio possibly constitutes an earlier diverging clade in comparison to the other group members.

Ten CSIs identified in this work are exclusively shared by members of the subgroup "Vinckeia" comprising of the following four genome-sequenced species P. berghei, P. chabaudi, P. vinckei and P. yoelii. One example of a CSIs specific for this clade is provided in Figure 5C. In this case, in a highly conserved region of the mitochondrial ribosomal protein L17-2, a one aa insert is specifically present in all members of "Vinckeia" group of species, but not in any other Plasmodium or Apicomplexa species. More detailed sequence information for this CSI and 9 other CSIs that are also specific for the "Vinckeia" clade is provided in Figures S51-S60 and their main characteristics are summarized in Table 3. Similarly, we have also identified eight CSIs that are specific for the "Plasmodium" subclade consisting of the following genome sequenced species P. vivax, P. gonderi, P. fragile, P. inui, P. knowlesi, 
P. coatneyi and P. cynomolgi. One example of a CSI consisting of a 5 aa insertion found in the protein leucine aminopeptidase, which is specific for this group is shown in Figure 5D. More detailed sequence information for this CSI as well as seven other CSIs which are also specifically found in all members of the "Plasmodium" clade is presented in Figures S41 and S60-S66 and information for these CSIs is summarized in Table 3.

\subsection{Molecular Signatures for the Mammalian-Infecting Plasmodium Species Clarifying their Evolutionary Relationships}

In addition to the CSIs that are specific for the genus Plasmodium and its four main subgenera, our analysis has also identified several CSIs that are helpful in clarifying the evolutionary relationships among the mammalian-infecting Plasmodium species, including P. malariae and P. ovale, whose association with the other subgenera is uncertain $[23,27-29,62]$. Currently both these species are taxonomically classified within the subgenus Plasmodium [17]. Of these other CSIs, four CSIs identified by our analysis are specifically shared by all Plasmodium species except those from "Haemamoeba" and "Laverania" clades. One example of a CSI exhibiting this kind of specificity is presented in Figure 6A. In this case, in the sequence alignment of the protein biotin-acetyl-CoA-carboxylase ligase protein, in a conserved region, a one aa insertion is commonly and exclusively shared by all available homologs from the "Plasmodium" and "Vinckeia" clades as well as P. malariae and P. ovale, but this CSI is absent in all other Plasmodium or Apicomplexa species. We refer to the group demarcated by these CSIs as the "Vinckeia-Plasmodium" clade. More detailed sequence information for the CSI shown in Figure 6A and three other CSIs which also exhibit similar specificities is provided in Figures S67-S70 and some of their characteristics are summarized in Table 3. The specific presence of these CSIs in members of the subgenera Plasmodium and Vinckeia as well as P. malariae and P. ovale, strongly suggests that these species shared a common ancestor exclusive of the other Plasmodium species, and the genetic changes responsible for these CSIs occurred in a common ancestor of this group after its divergence from other Plasmodium species.

The present study has also identified three CSIs that are exclusively shared by members of the "Plasmodium" clade and P. malariae, but not in any other Plasmodium or Apicomplexa species (the clade demarcated by these CSIs will be referred to here as the "Plasmodium-Malariae" clade). An example of a CSI consisting of a two aa insertion in the protein ubiquitin-activating enzyme E1, which is specifically shared by the above groups of species is shown in Figure 6B. Detailed sequence information for this CSI as well as two other CSIs exhibiting similar specificities is provided in Figures S70-S72 and some of their characteristics are summarized in Table 3. The unique shared presence of these CSIs in members of the subgenus Plasmodium and P. malariae strongly indicates that P. malariae is specifically related to the species which are part of this subgenus.

Lastly, we also report here identification of 3 CSIs that are specifically shared by members of the subgenus Vinckeia and P. ovale but not by any other Plasmodium or Apicomplexa species (this grouping will be referred to here as the "Vinckeia-Ovale" clade). An example of a CSI that is exclusively shared by these species is presented in Figure $6 \mathrm{C}$. In this instance, this figure shows partial sequence alignment of the protein phosphoinositide-specific phospholipase $\mathrm{C}$, where a one aa insertion is uniquely present in all members of the subgenus Vinckeia as well as in P. ovale, but not in any other species.

A more detailed description of this CSI and the two other CSIs exhibiting similar specificities is provided in Figures S73-S75 and information for them is summarized in Table 3. These three CSIs strongly indicate a close and specific relationship of P. ovale to members of the subgenus Vinckeia. A specific relationship between P. ovale and rodent-infecting Plasmodium species has also been observed in earlier work [27]. 


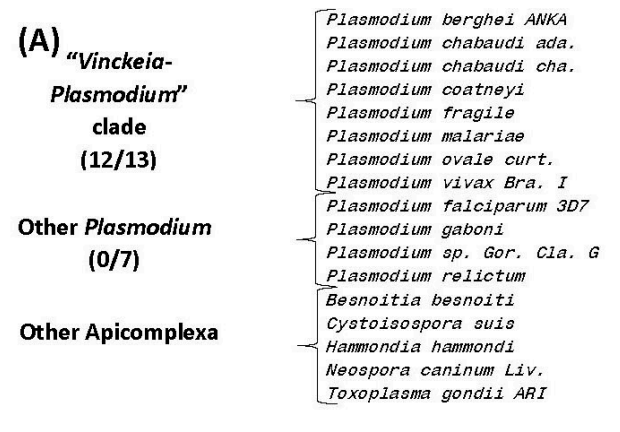

(B)

"Plasmodium-
$\begin{gathered}\text { Malariae" clade } \\ (7 / 8)\end{gathered}$
$\begin{gathered}\text { Other Plasmodium } \\ (0 / 12)\end{gathered}$
Other Apicom plexa

(C)

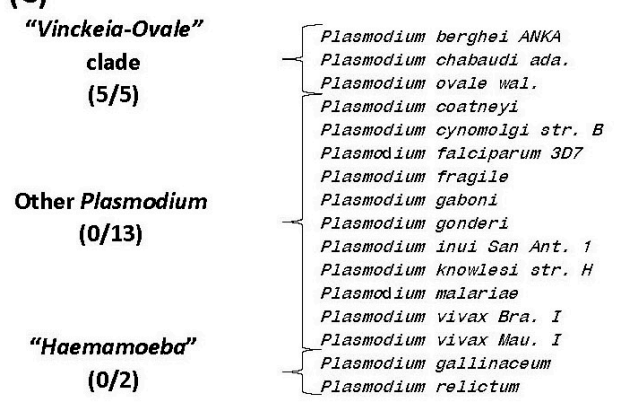

68069783
1061790962
1061786145
1139860911
817746710
1037133606
1036549794
901873339
258549083
1370972970
1370962886
1102622983
1261478615
1268232116
675134520
401402057
1005157216

901876239 457877603 672187706 1070256161 1037146568 1068927661 669195630 564280364 1269290753 1269290753
1103666945 1103660945 124806199
1370968857 1370968857 1370978736 1005157145 675134598 401402305
1098423783

1269288476 1061793446 1037150332 1139862609 457870197 258597029 817744384 1084858816 1194443604 672192942 1047805107 1037140543 901872403 901872403 901881473
1103666210 1102623540
28

68

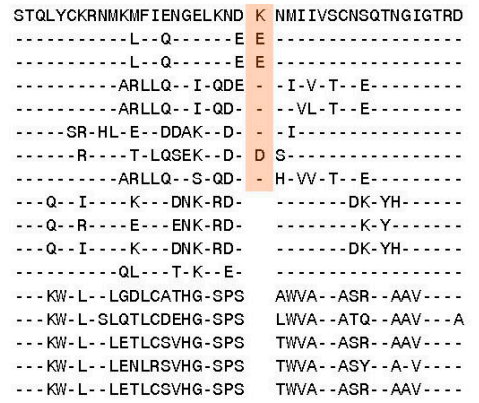

164 204 SLLVKYSKMVRSIS PV KKIAFLCCN IYGLCGYLFVDFGKGF

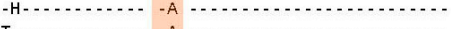
ENIL- -NN - I - - $-E$ NK DVIL- -N-L-N-N-Q T -DII- -NNL--G-E T $-N-\ldots-$ - S- V.

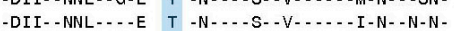

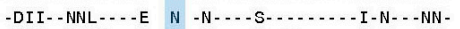

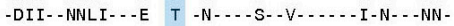
NDI I - NNL - . - K T T . . ED-I-NN-I-G-D K - R-...SED-I-NN - I- - D $\quad$ K ED-I- NN - I- . D K ...... EE-K-TNAFC- -A- T - PVG- IAA-VF- -AASV-..-L-EREE-K-NAFC- - A- - PVG-VAA-VF- -AASV-...-EREE-KL-NRFC-AAP RPVG-VAT-VF-AASI-..-ER -

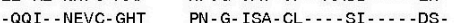

198238

FGLMYYHYKLVVNKSSR E RKKLKPFIYESNKLYDNYI ISGLK

. C...-Q--FI--G-G K-RM-S--G-G-............... -..-Q--FI-G-G K--M-S-...

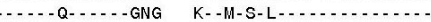
D...-Q-AI--G-G K--M-S-.

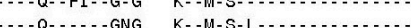

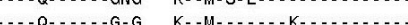

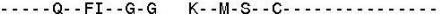
D.-.-Q-FI--G-G K-PM-S- C-G-....-Q-..C--G-G K- $-M N-$. -...-Q-OFI--G-G K-M-S--C K-

Figure 6. Examples of CSIs specific for the mammalian Plasmodium groups clarifying the genetic association of $P$. ovale and $P$. malariae species (A) Partial sequence alignment of the protein biotin-acetyl-CoA-carboxylase ligase showing a 1-aa insertion that is exclusively shared by all species from the subgenera Plasmodium and Vinckeia. (B) Partial sequence alignment of the ubiquitin-activating enzyme E1 protein showing a 2-aa insertion that is uniquely shared by different members of the subgenus Plasmodium but lacking in P. ovale. P. knowlesi contains a three aa insert in this position (not shown). (C) Partial sequence alignment of the phosphoinositide-specific phospholipase C protein showing a 1-aa insertion which is exclusively shared by members of the subgenus Vinckeia and P. ovale. Sequence information for multiple other CSIs exhibiting similar specificity is presented in Figures S67-S75 and their main characteristics are summarized in Table 3.

\subsection{Localizations of the CSIs in Protein Structures}

Earlier work on CSIs in protein sequences show that most, if not all, of the previously studied CSIs are located on the surface exposed loops of different proteins $[60,65]$. The surface exposed loops are known to play important roles in mediating novel protein-protein or protein-ligand interaction $[60,66,67]$. In view of these earlier studies, it was of interest to determine the locations of some of the Plasmodium-specific CSIs in the structures of proteins. In this regard, we have determined the structural locations of the CSIs in two proteins, 40S ribosomal protein S3 containing a one aa insertion specific for all Plasmodium (Figure 4A) and the protein leucine aminopeptidase, which contains a five aa insertion specific for subgenus "Plasmodium" (Figure 5D). The structure of the 40S ribosomal protein S3, which provides an important target for the binding of several drugs (such as 
protein synthesis inhibitor emetine) is available from Plasmodium species as well as other Apicomplexa species [58]. In Figure 7A we show the structural overlap of a homology model of the 40S ribosomal protein S3 from P. falciparum (colored in green) with the resolved structure of the homologous protein from T. gondii (colored in cyan). As seen from the structural overlap of the two proteins and a close up of the CSI region of the protein (Figure 7A), where the CSI is shown in red, the inserted phenylalanine residue is present on the surface of the protein and it leads to lengthening of a surface exposed $\alpha$ helix.

(A)

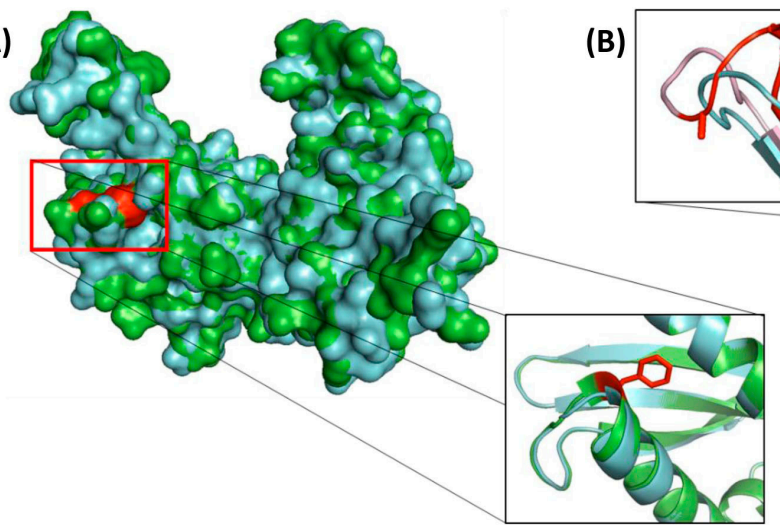

(B)

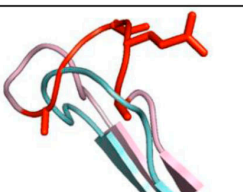

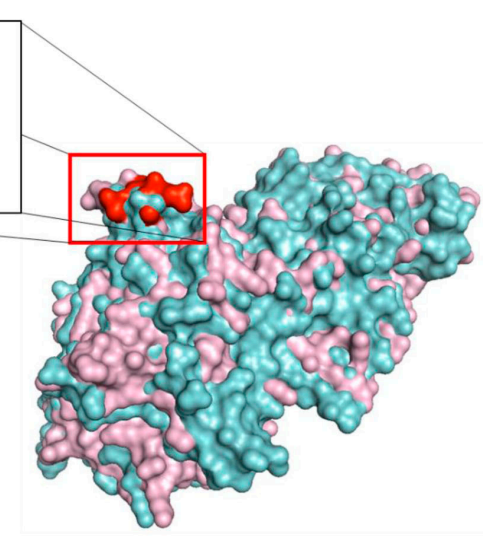

Figure 7. Structural localization of the identified CSIs in two of the studied proteins. (A) Structural overlap of a homology model of the 40 S ribosomal protein S3 from P. falciparum containing (shown in green) a 1 aa insertion specific for the genus Plasmodium (shown in Figure 4A) with the solved structure of the homologous protein from T. gondii (PDB ascension: 5XXU_D) lacking the CSI (shown in cyan). The CSI in the protein shown in red is present in a surface-exposed loop. (B) Structural overlap of a homology model of the protein leucine aminopeptidase from $P$. vivax containing a 5 aa insertion (see Figure 5D) (shown in pink) with the solved structure of the protein from P. falciparum (PDB ascension: 4ZX8_A) (shown in cyan). The five aa CSI is highlighted in red and it is present on the surface of the protein. As shown in the close up, it extends the length of an existing loop.

To examine the structural location of the CSI in leucine aminopeptidase protein (Figure 5D) a homology model of this protein from P. vivax was constructed as described in the Methods section. This model contains a five aa insertion which is specific for the "Plasmodium" clade (see Figure 5D for partial sequence alignment). Figure 7B shows a structural overlap of the homology model for P. vivax protein (colored pink) with the resolved structure of this protein form P. falciparum (colored in cyan). As seen, the residues corresponding to this CSI are also present in a surface loop lengthening this region of the protein.

\section{Discussion}

The class Hematozoa harbors several clinically important genera including the genus Plasmodium, which is responsible for the widely-prevalent and major life-threating disease malaria $[1,3,68,69]$. Hence, a good understanding of the interrelationships among different organisms comprising this class and reliable means for distinguishing them are of much importance [37]. In recent years, phylogenetic studies based on different gene/protein sequences have significantly advanced our understanding of the interrelationships among the hematozoa species $[3,8,10,16,19,20]$. However, due to the tight clustering of Plasmodium species in phylogenetic trees and the dependence of branching in phylogenetic trees on a large numbers of variables $[44,70,71]$, several relationships among the Plasmodium species remain ambiguous $[12,27,37]$. Hence, it is important to evaluate and confirm the interrelationships among Hematozoa/Plasmodium species by means of other sequence-based approaches that can provide more reliable information in these regards $[37,41,43,44,47,50]$.

The present study reports detailed phylogenetic and comparative genomic analyses on protein sequences from 28 genome-sequenced hematozoa species to understand their interrelationships. 
Phylogenetic trees were constructed based on two independent datasets of protein sequences comprising of either 14 transcription-translation related proteins or 10 metabolism-related proteins. Both these trees showed very similar branching pattern and they support a number of previously observed relationships. These include: (i) Distinct branching of the Piroplasmida species from the genus Plasmodium; (ii) Distant branching of B. microti, the causative agent of human babesiosis [6], from other members of the genus Babesia; (iii) Observance of three clades within the genus Plasmodium, "Haemamoeba", "Laverania" and "Vinckeia", which correspond to the similarly named subgenera. Additionally, a clade corresponding to the subgenus Plasmodium, (referred to as "Plasmodium" in the present work) was also observed but the species $P$. ovale and $P$. malariae, which according to the current classification are also part of this subgenus [17], did not group within this clade or showed any specific association with any of the other observed clades. Additionally, in the constructed phylogenetic trees, while there was a clear separation of the Piroplasmida clade from Plasmodium, the latter species formed a tight cluster and their interrelationships were not reliably resolved.

In view of these limitations of the phylogenetic tree construction approaches, a major focus of this work was on identifying CSIs that are uniquely shared by specific members of the class Hematozoa and using these characteristics for independently assessing the interrelationships among these species. As noted earlier, the CSIs represent an important class of molecular synapomorphies for reliable identification/demarcation of different monophyletic clades of organisms and assessing their interrelationships $[40,41,43,44,47,50]$. Extensive earlier work on these markers show that the relationships based on these rare genetic changes are minimally (and generally not) affected by different factors which confound inferences from phylogenetic approaches [44,50]. As a result, these markers have proven instrumental in resolving a number of important relationships which could not be resolved by phylogenetic approaches $[40,41,43,47]$. In cases where multiple CSIs supporting a given relationship are found, each CSI, present in a different gene/protein, provides independent evidence supporting the observed relationship. The present work has identified 79 CSIs that are exclusively present in specific groups of Hematozoa/Plasmodium species. A summary diagram showing the group-specificities of these CSIs is presented in Figure 8. It should be noted that in contrast to these CSIs, which are specific for the described groups/clades of species, our analysis has not identified any other reliable CSI(s) contradicting these relationships. In a few cases, as noted in the footnotes to the Tables and figure legends, a particular CSI in addition to being shared by different members of a given group was also present in an isolated species from another group. However, such cases were limited and showed no specific pattern or relationship. Further, for a number of Plasmodium species viz. P. falciparum, P. chabaudi, P. ovale, P. yoelii, P. vinckei and P. vivax, sequence information was available from a number of different strains. In these cases, as shown in the Supplementary Figures, the CSIs encompassing these species were present in all of the strains from these species and no intra-species variation in this regard was observed.

In this work (Figure 8) we have identified six CSIs that are exclusively found in all hematozoa species, two CSIs which are unique to members of the order Piroplasmida, five CSIs that are commonly shared by different Theileria and Babesia species except B. microti, and two CSIs which are specific for the genus Theileria. Additionally, large numbers of CSIs are specific for different members of the genus Plasmodium. Of these, 23 CSIs are distinctive characteristics of all Plasmodium species and two, nine, ten and eight CSIs are specifically shared by members of the subgenera "Haemamoeba", "Laverania", "Vinckeia" and "Plasmodium" (excepting P. ovale and P. malariae), respectively. Due to the exclusivity of the identified CSIs for the species from these clades, they provide novel and reliable molecular means for the demarcation of genus Plasmodium and a number of important groupings within it in more definitive terms.

In addition to the CSIs which are specific for different clades that are observed in phylogenetic trees, our analysis has also identified several other CSIs which support species relationships that are not evident from our trees. Of these CSIs, one CSI in the protein cysteine-tRNA ligase is uniquely shared by all mammalian-infecting Plasmodium species, but absent in the two avian infecting species 
("Haemamoeba") as well as all other Apicomplexa species. The absence of this CSI in the outgroup species (Apicomplexa as well as other eukaryotic organisms) indicates that this CSI is an insert and the genetic change responsible for this insertion occurred in a common ancestor of the mammalian-infecting Plasmodium species after this group diverged from other Apicomplexa-Plasmodium species. Although there was only one CSI identified of this kind, due to its high degree of conservation and the presence of this protein in different Apicomplexa species as well as other eukaryotic organisms, this CSI constitutes a reliable molecular characteristic. The species distribution of this CSI provides molecular evidence that the avian-infecting Plasmodium species are ancestral to the mammalian-infecting group of species. Although, the avian origin of mammalian-infecting species Plasmodium species has been suggested previously [39,64], this inference was not supported by other studies $[3,12,37,72,73]$.

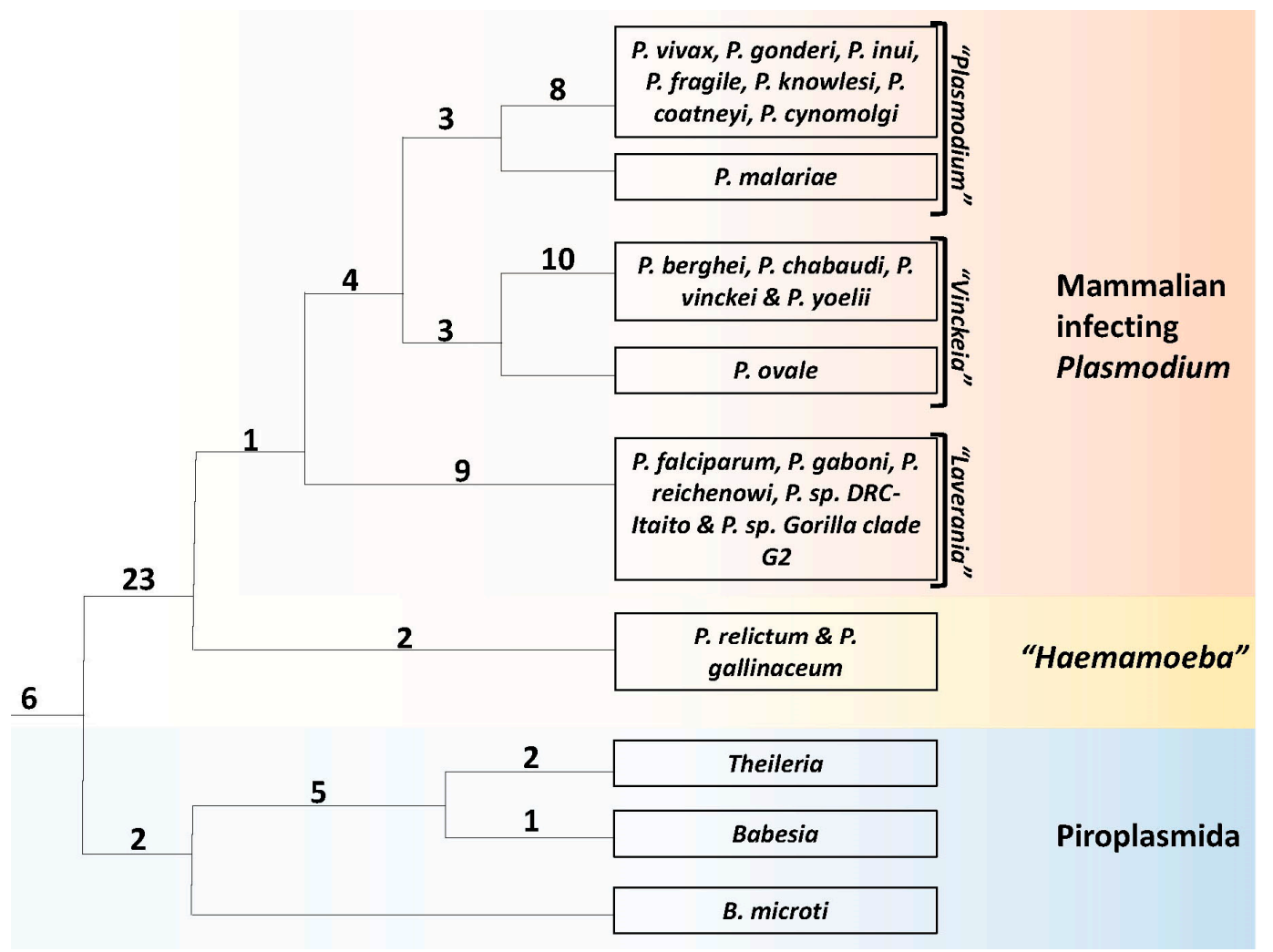

Figure 8. A conceptual diagram summarizing the evolutionary relationships among members of the class Hematozoa and the genus Plasmodium based on phylogenetic analysis and different identified molecular signatures (synapomorphies). The numbers of CSIs that are specific for different clades or species-groupings are noted on the respective nodes.

Another important inference supported by CSIs, which is not evident from phylogenetic trees, is a specific relationship of the species from the subgenera Plasmodium and Vinckeia (including P. ovale and P. malariae). The shared presences of four CSIs in 4 different proteins that are uniquely found in the members of these two subgenera provide strong evidence that the species from these two subgenera are specifically related and that they shared a common ancestor exclusive of the other Plasmodium-Apicomplexa species. Further, the absence of these CSIs in the outgroup species (other Apicomplexa species or eukaryotic organisms), strongly suggests that these two subgenera of Plasmodium have diverged subsequent to the branching/evolution of species from the subgenera Haemamoeba and Laverania, which lack these CSIs. These results indicate that the Plasmodium species infecting other mammals have originated after the divergence (evolution) of avian- and great apes-infecting Plasmodium species. 
Several other CSIs identified in this work serve to clarify the evolutionary relationships of P. ovale and P. malariae, which do not group specifically with any of the four observed clades of Plasmodium species seen in the phylogenetic trees (Figure 1). The phylogenetic affiliation of these two species, which although are placed in the subgenus Plasmodium, is found to be highly variable and not resolved by earlier studies $[12,23,27-29,62]$. In this context, our identification of three CSIs, which are uniquely shared by P. malariae as well as by different species from the subgenus Plasmodium (except P. ovale), provides reliable evidence that $P$. malariae is specifically related to the subgenus Plasmodium (except P. ovale) and it shared a common ancestor with this group exclusive of the other Plasmodium genus species including P. ovale. Similarly, the three CSIs identified in other proteins, which are exclusively shared by P. ovale and different members of the subgenus Vinckeia, support a specific association of this species with this latter subgenus. It should be noted that while the branching of $P$. malariae with members of the subgenus Plasmodium and an association of P. ovale with Vinckeia subgenus has been observed in some earlier studies [12,23,27-29,62], it is for the first time that based on the identification of multiple CSIs, we are able to reliably show in the same study the specific associations of P. malariae and P. ovale with the subgenera Plasmodium and Vinckeia, respectively. These results indicate that P. ovale should be moved from the subgenus Plasmodium to the subgenus Vinckeia.

Comprehensive analysis of the hematozoa/Plasmodium genomes presented in this study has enabled us to develop a reliable framework for understanding the evolutionary relationships among these organisms. Although our analysis is limited by the number of genome sequences currently available for the species from this class/genus, extensive earlier work on CSIs for other groups of organisms strongly indicate that these molecular characteristics exhibit a high degree constancy and predictive ability to be found in other members of the indicated groups $[40,44,49,51]$. Hence, they provide us important means for clarifying the evolutionary relationships of other species related to these groups and for taxonomic studies [44,46,52]. A recent comprehensive study which has examined a broad range of Plasmodium species from diverse vertebrate hosts has suggested that the genus Plasmodium may be polyphyletic [37]. It will be of much interest to examine the presence/absence of some of the described CSIs, which are specific for the genus Plasmodium, in these studied species to further confirm this inference and to investigate the relationships of species within this genus.

Members of the genus Plasmodium, particularly the species P. falciparum, are the primary causative agents of the highly life-threating disease malaria $[3,4,68,69,74]$. Malaria is widely prevalent in Sub-Saharan Africa, Asia, and Latin America and in the year 2016 alone, 216 million people were infected with the disease leading to over 400,000 deaths $[13,68,69,75]$. As infections with Plasmodium species differ greatly in terms of the symptoms and severity of the disease, accurate identification of the causative species in the infected individual is of major importance. Diagnosis of malaria is currently based primarily on microscopic examination of the blood for infective organism or antigen-based tests [76]. However, it is of much importance to develop other rapid and reliable tests that can accurately identify the infective species. The molecular markers identified in the present study, which are specific for the genus Plasmodium as well as its different subgroups (subgenera), due to their presence in conserved regions and exclusivity for these groups, provide important means for developing novel and reliable diagnostic methods for the identification of different groups of Plasmodium species. Based on the sequence regions encompassing these CSIs, novel diagnostic tests can be developed by means of different commonly used techniques such as PCR-based, q-PCR-based, pyrosequencing, immunological or antibody-based methods, MALDI-TOF, aptamer-based methods, as well as rapid in silico identification of the CSI-containing organisms in genomic and metagenomic sequences by means of BLAST searches. In earlier work, the CSIs have been used for developing novel and highly-specific diagnostic tests for the important bacterial pathogens Bacillus anthracis and Escherichia coli O157/H7 [77,78].

A large number of the CSIs identified in this work are found in important proteins which carry out essential functions in Apicomplexa as well as other eukaryotic organisms. Although the cellular functions of these CSIs are currently not known, earlier work on CSIs in other organisms has shown 
that these molecular characteristics are essential or play important functional roles in the organisms where they are found $[60,79,80]$. The CSIs in protein sequences are generally found in the protein surface loops, which are indicated to play important roles in mediating novel protein-protein or protein-ligand interactions that are essential or important for the CSI-containing organisms [60,66,80]. The Plasmodium-specific CSIs in the structures of two proteins i.e., $40 \mathrm{~S}$ ribosomal protein S3 and the leucine aminopeptidase protein, which were studied in this regard, are also both found in the surface-exposed loops of these proteins. In view of the specificity of these CSIs for Plasmodium or Hematozoa species, it is of much interest to determine the cellular functions of these novel molecular characteristics. Such studies could lead to discovery of novel biochemical and/or other properties that are specific and important for different groups of organisms within the class Hematozoa and the genus Plasmodium.

Development of resistance to existing drugs in $P$. falciparum poses one of the greatest threats in the control and treatment of malaria $[68,75,81,82]$. Thus, for protecting human populations from malaria, particularly in malaria endemic countries, development of new drugs that are effective in the treatment/control of malaria is a top public health priority. Therefore, identification of potential targets that could be exploited for development of new antimalarial drugs is of much importance. In this context, it is important to point out that the CSIs in protein sequences exhibit a number of characteristics, which make them potentially useful targets for development of a new class of therapeutics [83,84]. The potential usefulness of the CSIs as drug targets stems from a number of key observations: (i) they exhibit high degree of specificity for a given group of organism (e.g., Plasmodium); (ii) earlier work on CSIs strongly suggests that these molecular characteristics play important/essential functions in the CSI-containing organisms [80]; (iii) The CSIs in protein sequences, as demonstrated for the two CSIs examined in this study, are located in surface-exposed loops of the proteins, which are implicated in mediating novel protein-protein or protein-ligand interactions that are important for the CSI-containing organisms; (iv) Homologs of the CSI-containing proteins, or the CSIs in such homologs, are generally not found in humans. Based on these characteristics, one expects that screening for compounds, which bind specifically to the CSIs (or CSI- containing regions) and thereby inhibit the cellular functions of the CSIs could lead to the discovery of a novel class of drugs that will specifically target the Plasmodium species. Thus, the molecular markers identified in the present study, in addition to providing a reliable framework for understanding the evolutionary relationships among the Plasmodium species, provide novel means for exploring several important aspects of these clinically important parasitic organisms.

Supplementary Materials: The following are available online at http://www.mdpi.com/2073-4425/10/7/490/s1, Table S1. Some characteristics of the Hematozoa genomes used in Phylogenetic/Comparative Genomic Studies. Table S2. P. falciparum protein sequences utilized to construct the tree shown in Figure 1A. Table S3. P. falciparum protein sequences utilized to construct the tree shown in Figure 1B. Table S4. Summary of some characteristics of Plasmodium species featured in Figure 1. Figure S1. Partial sequence alignment of Cell division cycle protein 48 homologue showing a Hematozoa specific CSI. Figure S2. Partial sequence alignment of 30S ribosomal protein S9 showing a Hematozoa specific CSI. Figure S3. Partial sequence alignment of 40S ribosomal protein S12 showing a Hematozoa specific CSI. Figure S4. Partial sequence alignment of $20 \mathrm{~S}$ proteasome $\beta 4$ subunit protein showing a Hematozoa specific CSI. Figure S5. Partial sequence alignment of Golgi reassembly-stacking protein 1 showing a Hematozoa specific CSI. Figure S6. Partial sequence alignment of Pyruvate kinase 2 protein showing a Hematozoa specific CSI. Figure S7. Partial sequence alignment of Succinyl-CoA synthetase $\beta$ chain protein showing a Piroplasmida specific CSI. Figure S8. Partial sequence alignment of a Hypothetical protein showing a Piroplasmida specific CSI. Figure S9. Partial sequence alignment of Dihydrolipoamide dehydrogenase protein showing a Piroplasmida (except B. microti) specific CSI. Figure S10. Partial sequence alignment of a Hypothetical protein showing a Piroplasmida (except B. microti) specific CSI. Figure S11. Partial sequence alignment of a Hypothetical protein showing a Piroplasmida (except B. microti) specific CSI. Figure S12. Partial sequence alignment of Intron-binding aquarius $\beta$ like protein showing a Piroplasmida (excluding B. microti) specific CSI. Figure S13. Partial sequence alignment of Intron-binding aquarius $\beta$ like protein showing a Piroplasmida (excluding B. microti) specific CSI. Figure S14. Partial sequence alignment of Cysteinyl-tRNA synthetase protein showing a Babesia (excluding B. microti) specific CSI and a Theileria specific CSI. Figure S15. Partial sequence alignment of Eukaryotic translation initiation factor 4a protein showing a Theileria specific CSI. Figure S16. Partial sequence alignment of $40 S$ ribosomal protein S3 showing a Plasmodium specific CSI. Figure S17. Partial sequence alignment of $26 \mathrm{~S}$ proteasome regulatory subunit RPN2 protein showing a Plasmodium specific CSI. Figure S18. Partial sequence alignment of $26 \mathrm{~S}$ proteasome regulatory subunit 4 protein showing a Plasmodium specific CSI. Figure S19. Partial sequence alignment of 40 S ribosomal protein S25 showing a Plasmodium specific CSI. Figure 
S20. Partial sequence alignment of 50 S ribosomal protein L1, mitochondrial showing a Plasmodium specific CSI. Figure S21. Partial sequence alignment of 60 S ribosomal protein L35 showing a Plasmodium specific CSI. Figure S22. Partial sequence alignment of Asparagine-tRNA ligase protein showing a Plasmodium specific CSI. Figure S23. Partial sequence alignment of ATP-dependent RNA helicase DBP10 protein showing a Plasmodium specific CSI. Figure S24. Partial sequence alignment of Alternative splicing regulator protein showing a Plasmodium specific CSI. Figure S25. Partial sequence alignment of Adenosinetriphosphatase protein showing a Plasmodium specific CSI. Figure S26. Partial sequence alignment of DNA2/NAM7 helicase protein showing a Plasmodium specific CSI. Figure S27. Partial sequence alignment of Elongation factor Tu family protein showing a Plasmodium specific CSI. Figure S28. Partial sequence alignment of Multidrug resistance protein 2 showing a Plasmodium specific CSI. Figure S29. Partial sequence alignment of Pre-mRNA-processing-splicing factor 8 protein showing a Plasmodium specific CSI. Figure S30. Partial sequence alignment of Pyruvate dehydrogenase E1 component, $\alpha$ subunit protein showing a Plasmodium specific CSI. Figure S31. Partial sequence alignment of Pyruvate dehydrogenase E1 component, $\alpha$ subunit protein showing a Plasmodium specific CSI. Figure S32. Partial sequence alignment of Ras-related protein Rab-5A showing a Plasmodium specific CSI. Figure S33. Partial sequence alignment of Ribosomal protein L27a showing a Plasmodium specific CSI. Figure S34. Partial sequence alignment of RuvB-like helicase isoform 1 protein showing a Plasmodium specific CSI. Figure S35. Partial sequence alignment of RuvB-like helicase isoform 1 protein showing a Plasmodium specific CSI. Figure S36. Partial sequence alignment of RuvB-like helicase isoform 2 protein showing a Plasmodium specific CSI. Figure S37. Partial sequence alignment of Splicing factor 3A subunit 2 protein showing a Plasmodium specific CSI. Figure S38. Partial sequence alignment of Translation initiation factor SUI1 protein showing a Plasmodium specific CSI. Figure S39. Partial sequence alignment of Cysteine-tRNA ligase protein showing a Mammalian infecting clades specific CSI. Figure S40. Partial sequence alignment of Protein phosphatase protein showing a subgenus "Haemamoeba" specific CSI. Figure S41. Partial sequence alignment of Conserved Plasmodium protein showing a subgenus "Haemamoeba" specific CSI and a subgenus "Plasmodium" specific CSI. Figure S42. Partial sequence alignment of Eukaryotic translation initiation factor 3 subunit D protein showing a subgenus "Laverania" specific CSI. Figure S43. Partial sequence alignment of Conserved hypothetical protein showing a subgenus "Laverania" specific CSI. Figure S44. Partial sequence alignment of Aconitate hydratase protein showing a subgenus "Laverania" specific CSI. Figure S45. Partial sequence alignment of Conserved Plasmodium protein showing a subgenus "Laverania" specific CSI. Figure S46. Partial sequence alignment of Cation-transporting ATPase protein showing a subgenus "Laverania" specific CSI. Figure S47. Partial sequence alignment of Serine/threonine protein kinase showing a subgenus "Laverania" specific CSI. Figure S48. Partial sequence alignment of Tetratricopeptide repeat family protein showing a subgenus "Laverania" specific CSI. Figure S49. Partial sequence alignment of Thioredoxin-like protein showing a subgenus "Laverania" specific CSI. Figure S50. Partial sequence alignment of Pre-mRNA-processing-splicing factor 8 protein showing a subgenus "Laverania" specific CSI. Figure S51. Partial sequence alignment of Mitochondrial ribosomal protein L17-2 precursor protein showing a subgenus "Vinckeia" specific CSI. Figure S52. Partial sequence alignment of Gdp-mannose 4,6 dehydratase protein showing a subgenus "Vinckeia" specific CSI. Figure S53. Partial sequence alignment of 14-3-3 protein showing a subgenus "Vinckeia" specific CSI. Figure S54. Partial sequence alignment of Conserved Plasmodium protein showing a subgenus "Vinckeia" specific CSI. Figure S55. Partial sequence alignment of Conserved Plasmodium protein showing a subgenus "Vinckeia" specific CSI. Figure S56. Partial sequence alignment of Conserved Plasmodium protein showing a subgenus "Vinckeia" specific CSI. Figure S57. Partial sequence alignment of M17 leucyl aminopeptidase protein showing a subgenus "Vinckeia" specific CSI. Figure S58. Partial sequence alignment of PelOta protein homologue showing a subgenus "Vinckeia" specific CSI. Figure S59. Partial sequence alignment of LCCL domain-containing protein showing a subgenus "Vinckeia" specific CSI. Figure S60. Partial sequence alignment of DEAD-box family helicase 4 protein showing a subgenus "Vinckeia" specific CSI and a subgenus "Plasmodium" specific CSI. Figure S61. Partial sequence alignment of Leucine aminopeptidase protein showing a subgenus "Plasmodium" specific CSI. Figure S62. Partial sequence alignment of Conserved hypothetical protein showing a subgenus "Plasmodium" specific CSI. Figure S63. Partial sequence alignment of Hypothetical protein PVBG_03892 showing a subgenus "Plasmodium" specific CSI. Figure S64. Partial sequence alignment of Hypothetical protein PVMG_00581 showing a subgenus "Plasmodium" specific CSI. Figure S65. Partial sequence alignment of Hypothetical protein PVNG_02680 showing a subgenus "Plasmodium" specific CSI. Figure S66 Partial sequence alignment of Serine/threonine protein kinase showing a subgenus "Plasmodium" specific CSI. Figure S67. Partial sequence alignment of Biotin-acetyl-CoA-carboxylase ligase protein showing a "Vinckeia-Plasmodium" clade specific CSI. Figure S68. Partial sequence alignment of Hypothetical protein PVIIG_05030 showing a "Vinckeia-Plasmodium" clade specific CSI. Figure S69. Partial sequence alignment of Conserved Plasmodium protein showing a "Vinckeia-Plasmodium" clade specific CSI. Figure S70. Partial sequence alignment of Conserved Plasmodium protein showing a "Vinckeia-Plasmodium" clade specific CSI and a "Plasmodium-Malariae" clade specific CSI. Figure S71. Partial sequence alignment of Ubiquitin-activating enzyme E1 protein showing a "Plasmodium-Malariae" clade specific CSI. Figure S72. Partial sequence alignment of Hypothetical protein PVNG_01558 showing a "Plasmodium-Malariae" clade specific CSI. Figure S73. Partial sequence alignment of Phosphoinositide-specific phospholipase C protein showing a "Vinckeia-Ovale" clade specific CSI. Figure S74. Partial sequence alignment of DEAD/DEAH helicase protein showing a "Vinckeia-Ovale" clade specific CSI. Figure S75. Partial sequence alignment of ATPase protein showing a "Vinckeia-Ovale" clade specific CSI. 
Author Contributions: R.S.: Identification of CSIs and confirming their species specificities, phylogenetic analysis, homology modelling and localization of the CSIs in protein structures, preparation of draft manuscript; R.S.G.: Planning and supervision of the entire work, Creation of sequence alignments of the proteins for identification of the CSIs, Interpretation of the results and writing and finalizing of the manuscript, obtained funding for the project.

Acknowledgments: This work was supported by Research Grant number 249924 from the Natural Science and Engineering Research Council of Canada awarded to Radhey S. Gupta.

Conflicts of Interest: The authors declare no conflict of interest.

\section{References}

1. Telford, S.R. The Hemoparasites of the Reptilia; CRC Press: Boca Raton, FL, USA, 2008.

2. Garnham, P.C. Malaria Parasites and Other Haemosporidia; Blackwell Scientific Publications: Oxford, UK, 1966.

3. Perkins, S.L. Malaria's many mates: Past, present, and future of the systematics of the order Haemosporida. J. Parasitol. 2014, 100, 11-25. [CrossRef] [PubMed]

4. Votypka, J.; Modry, D.; ObornÃ-k, M.; Slapeta, J.; LukeÅ, J. Apicomplexa. In Handbook of the Protists; Archibald, J., Simpson, A., Slamovits, C., Eds.; Springer: Cham, Switzerland, 2017; pp. 1-58.

5. Allsopp, M.T.; Allsopp, B.A. Molecular sequence evidence for the reclassification of some Babesia species. Ann. NY Acad. Sci. 2006, 1081, 509-517. [CrossRef] [PubMed]

6. Homer, M.J.; Aguilar-Delfin, I.; Telford, S.R.; Krause, P.J.; Persing, D.H. Babesiosis. Clin. Microbiol. Rev. 2000, 13, 451-469. [CrossRef] [PubMed]

7. Kamau, J.; de Vos, A.J.; Playford, M.; Salim, B.; Kinyanjui, P.; Sugimoto, C. Emergence of new types of Theileria orientalis in Australian cattle and possible cause of theileriosis outbreaks. Parasit. Vectors 2011, 4, 22. [CrossRef] [PubMed]

8. Lack, J.B.; Reichard, M.V.; Van Den Bussche, R.A. Phylogeny and evolution of the Piroplasmida as inferred from 18S rRNA sequences. Int. J. Parasitol. 2012, 42, 353-363. [CrossRef] [PubMed]

9. Wenyon, C.M. Protozoology: A Manual for Medical Men, Veterinarians and Zoologists; W. Wood: New York, NY, USA, 1926.

10. Schreeg, M.E.; Marr, H.S.; Tarigo, J.L.; Cohn, L.A.; Bird, D.M.; Scholl, E.H.; Levy, M.G.; Wiegmann, B.M.; Birkenheuer, A.J. Mitochondrial Genome Sequences and Structures Aid in the Resolution of Piroplasmida phylogeny. PLOS ONE 2016, 11, e0165702. [CrossRef]

11. Valkiunas, G. Avian Malaria Parasites and Other Haemosporidia; CRC Press: Boca Raton, FL, USA, 2005.

12. Arisue, N.; Hashimoto, T. Phylogeny and evolution of apicoplasts and apicomplexan parasites. Parasitol. Int. 2015, 64, 254-259. [CrossRef]

13. Hay, S.I.; Okiro, E.A.; Gething, P.W.; Patil, A.P.; Tatem, A.J.; Guerra, C.A.; Snow, R.W. Estimating the global clinical burden of Plasmodium falciparum malaria in 2007. PLoS Med. 2010, 7. [CrossRef]

14. Sachs, J.; Malaney, P. The economic and social burden of malaria. Nature 2002, 415, 680-685. [CrossRef]

15. Martinsen, E.S.; Paperna, I.; Schall, J.J. Morphological versus molecular identification of avian Haemosporidia: An exploration of three species concepts. Parasitology 2006, 133, 279-288. [CrossRef]

16. Martinsen, E.S.; Perkins, S.L.; Schall, J.J. A three-genome phylogeny of malaria parasites (Plasmodium and closely related genera): Evolution of life-history traits and host switches. Mol. Phylogenet. Evol. 2008, 47, 261-273. [CrossRef] [PubMed]

17. Telford, S.R.; Johnson, R.N.; Young, D.G. Additional Plasmodium species from Anolis lizards of Hispaniola and Panama. Int. J. Parasitol. 1989, 19, 275-284. [CrossRef]

18. Perkins, S.L.; Martinsen, E.S.; Falk, B.G. Do molecules matter more than morphology? Promises and pitfalls in parasites. Parasitology 2011, 138, 1664-1674. [CrossRef] [PubMed]

19. Borner, J.; Pick, C.; Thiede, J.; Kolawole, O.M.; Kingsley, M.T.; Schulze, J.; Cottontail, V.M.; Wellinghausen, N.; Schmidt-Chanasit, J.; Bruchhaus, I.; et al. Phylogeny of haemosporidian blood parasites revealed by a multi-gene approach. Mol. Phylogenet. Evol. 2016, 94, 221-231. [CrossRef] [PubMed]

20. Perkins, S.L.; Sarkar, I.N.; Carter, R. The phylogeny of rodent malaria parasites: Simultaneous analysis across three genomes. Infect. Genet. Evol. 2007, 7, 74-83. [CrossRef] [PubMed]

21. Honma, H.; Kawai, S.; Motooka, D.; Nakamura, S.; Tougan, T.; Horii, T.; Arisue, N. Draft Genome Sequence of Plasmodium gonderi, a Malaria Parasite of African Old World Monkeys. Genome Announc. 2017, 5. [CrossRef] [PubMed] 
22. Tachibana, S.; Sullivan, S.A.; Kawai, S.; Nakamura, S.; Kim, H.R.; Goto, N.; Arisue, N.; Palacpac, N.M.; Honma, H.; Yagi, M.; et al. Plasmodium cynomolgi genome sequences provide insight into Plasmodium vivax and the monkey malaria clade. Nat. Genet. 2012, 44, 1051-1055. [CrossRef] [PubMed]

23. Perkins, S.L.; Schall, J.J. A molecular phylogeny of malarial parasites recovered from cytochrome $b$ gene sequences. J. Parasitol. 2002, 88, 972-978. [CrossRef]

24. Ricklefs, R.E.; Fallon, S.M.; Bermingham, E. Evolutionary relationships, cospeciation, and host switching in avian malaria parasites. Syst. Biol. 2004, 53, 111-119. [CrossRef]

25. Dimitrov, D.; Zehtindjiev, P.; Bensch, S. Genetic diversity of avian blood parasites in SE Europe: Cytochrome $\mathrm{b}$ lineages of the genera Plasmodium and Haemoproteus (Haemosporida) from Bulgaria. Acta Parasitol. 2010, 55, 201-209. [CrossRef]

26. Bensch, S.; Hellgren, O.; Perez-Tris, J. MalAvi: A public database of malaria parasites and related haemosporidians in avian hosts based on mitochondrial cytochrome b lineages. Mol. Ecol. Resour. 2009, 9, 1353-1358. [CrossRef] [PubMed]

27. Arisue, N.; Hashimoto, T.; Mitsui, H.; Palacpac, N.M.; Kaneko, A.; Kawai, S.; Hasegawa, M.; Tanabe, K.; Horii, T. The Plasmodium apicoplast genome: Conserved structure and close relationship of P. ovale to rodent malaria parasites. Mol. Biol. Evol. 2012, 29, 2095-2099. [CrossRef] [PubMed]

28. Ansari, H.R.; Templeton, T.J.; Subudhi, A.K.; Ramaprasad, A.; Tang, J.; Lu, F.; Naeem, R.; Hashish, Y.; Oguike, M.C.; Benavente, E.D.; et al. Genome-scale comparison of expanded gene families in Plasmodium ovale wallikeri and Plasmodium ovale curtisi with Plasmodium malariae and with other Plasmodium species. Int. J. Parasitol. 2016, 46, 685-696. [CrossRef] [PubMed]

29. Rutledge, G.G.; Bohme, U.; Sanders, M.; Reid, A.J.; Cotton, J.A.; Maiga-Ascofare, O.; Djimde, A.A.; Apinjoh, T.O.; Amenga-Etego, L.; Manske, M.; et al. Plasmodium malariae and P. ovale genomes provide insights into malaria parasite evolution. Nature 2017, 542, 101-104. [CrossRef] [PubMed]

30. Barta, J.R.; Ogedengbe, J.D.; Martin, D.S.; Smith, T.G. Phylogenetic position of the adeleorinid coccidia (Myzozoa, Apicomplexa, Coccidia, Eucoccidiorida, Adeleorina) inferred using 18S rDNA sequences. J. Eukaryot. Microbiol. 2012, 59, 171-180. [CrossRef] [PubMed]

31. Weiner, J.; Kooij, T.W. Phylogenetic profiles of all membrane transport proteins of the malaria parasite highlight new drug targets. Microb. Cell 2016, 3, 511-521. [CrossRef] [PubMed]

32. Morrison, D.A.; Ellis, J.T. Effects of nucleotide sequence alignment on phylogeny estimation: A case study of 18S rDNAs of apicomplexa. Mol. Biol. Evol. 1997, 14, 428-441. [CrossRef] [PubMed]

33. Nakajima, R.; Tsuji, M.; Oda, K.; Zamoto-Niikura, A.; Wei, Q.; Kawabuchi-Kurata, T.; Nishida, A.; Ishihara, C. Babesia microti-group parasites compared phylogenetically by complete sequencing of the CCTeta gene in 36 isolates. J. Vet. Med. Sci. 2009, 71, 55-68. [CrossRef]

34. Corradetti, A.; Garnham, P.C.C.; Laird, M. New Classification of the Avian Malaria Parasites. Parassitologia 1963, 5, 1-4.

35. Bray, R.S. Studies on malaria in chimpanzees. VI. Laverania falciparum. Am. J. Trop. Med. Hyg. 1958, 7, 20-24. [CrossRef]

36. Garnham, P.C. The Subgenera of Plasmodium in mammals. Ann. Soc. Belges. Med. Trop. Parasitol. Mycol. 1964, 44, 267-271. [PubMed]

37. Galen, S.C.; Borner, J.; Martinsen, E.S.; Schaer, J.; Austin, C.C.; West, C.J.; Perkins, S.L. The polyphyly of Plasmodium: Comprehensive phylogenetic analyses of the malaria parasites (order Haemosporida) reveal widespread taxonomic conflict. R. Soc. Open. Sci. 2018, 5, 171780. [CrossRef] [PubMed]

38. Pick, C.; Ebersberger, I.; Spielmann, T.; Bruchhaus, I.; Burmester, T. Phylogenomic analyses of malaria parasites and evolution of their exported proteins. BMC Evol. Biol. 2011, 11, 167. [CrossRef] [PubMed]

39. Waters, A.P.; Higgins, D.G.; McCutchan, T.F. Plasmodium falciparum appears to have arisen as a result of lateral transfer between avian and human hosts. Proc. Natl. Acad. Sci. USA 1991, 88, 3140-3144. [CrossRef] [PubMed]

40. Gupta, R.S. Molecular signatures that are distinctive characteristics of the vertebrates and chordates and supporting a grouping of vertebrates with the tunicates. Mol. Phylogenet. Evol. 2016, 94, 383-391. [CrossRef] [PubMed]

41. Springer, M.S.; Stanhope, M.J.; Madsen, O.; de Jong, W.W. Molecules consolidate the placental mammal tree. Trends Ecol. Evol. 2004, 19, 430-438. [CrossRef] [PubMed] 
42. Rivera, M.C.; Lake, J.A. Evidence that eukaryotes and eocyte prokaryotes are immediate relatives. Science 1992, 257, 74-76. [CrossRef] [PubMed]

43. Baldauf, S.L.; Palmer, J.D. Animals and fungi are each other's closest relatives: Congruent evidence from multiple proteins. Proc. Natl. Acad. Sci. USA 1993, 90, 11558-11562. [CrossRef]

44. Gupta, R.S. Impact of genomics on the understanding of microbial evolution and classification: The importance of Darwin's views on classification. FEMS Microbiol. Rev. 2016, 40, 520-553. [CrossRef]

45. Gupta, R.S. Protein phylogenies and signature sequences: A reappraisal of evolutionary relationships among archaebacteria, eubacteria, and eukaryotes. Microbiol. Mol. Biol. Rev. 1998, 62, 1435-1491.

46. Gupta, R.S.; Lo, B.; Son, J. Phylogenomics and Comparative Genomic Studies Robustly Support Division of the Genus Mycobacterium into an Emended Genus Mycobacterium and Four Novel Genera. Front. Microbiol. 2018, 9, 67. [CrossRef] [PubMed]

47. Gupta, R.S. Identification of Conserved Indels that are Useful for Classification and Evolutionary Studies. In Methods in Microbiology, New Approaches to Prokaryotic Systematics; Goodfellow, M., Sutcliffe, I., Chun, J., Eds.; Academic Press: Cambridge, MA, USA, 2014; pp. 153-182.

48. Bapteste, E.; Philippe, H. The potential value of indels as phylogenetic markers: Position of trichomonads as a case study. Mol. Biol. Evol. 2002, 19, 972-977. [CrossRef] [PubMed]

49. Naushad, H.S.; Lee, B.; Gupta, R.S. Conserved signature indels and signature proteins as novel tools for understanding microbial phylogeny and systematics: Identification of molecular signatures that are specific for the phytopathogenic genera Dickeya, Pectobacterium and Brenneria. Int. J. Syst. Evol. Microbiol. 2014, 64, 366-383. [CrossRef] [PubMed]

50. Rokas, A.; Holland, P.W. Rare genomic changes as a tool for phylogenetics. Trends Ecol. Evol. 2000, 15, 454-459. [CrossRef]

51. Bhandari, V.; Naushad, H.S.; Gupta, R.S. Protein based molecular markers provide reliable means to understand prokaryotic phylogeny and support Darwinian mode of evolution. Front. Cell. Infect. Microbiol. 2012, 2, 98. [CrossRef]

52. Adeolu, M.; Alnajar, S.; Naushad, S.; Gupta, S. Genome-based phylogeny and taxonomy of the 'Enterobacteriales': Proposal for Enterobacterales ord. nov. divided into the families Enterobacteriaceae, Erwiniaceae fam. nov., Pectobacteriaceae fam. nov., Yersiniaceae fam. nov., Hafniaceae fam. nov., Morganellaceae fam. nov., and Budviciaceae fam. nov. Int. J. Syst. Evol. Microbiol. 2016, 66, 5575-5599.

53. Capella-Gutierrez, S.; Silla-Martinez, J.M.; Gabaldon, T. trimAl: A tool for automated alignment trimming in large-scale phylogenetic analyses. Bioinformatics 2009, 25, 1972-1973. [CrossRef]

54. Price, A.L.; Kryukov, G.V.; de Bakker, P.I.; Purcell, S.M.; Staples, J.; Wei, L.J.; Sunyaev, S.R. Pooled association tests for rare variants in exon-resequencing studies. Am. J. Hum. Genet. 2010, 86, 832-838. [CrossRef]

55. Stamatakis, A. RAxML version 8: A tool for phylogenetic analysis and post-analysis of large phylogenies. Bioinformatics 2014, 30, 1312-1313. [CrossRef]

56. Sievers, F.; Wilm, A.; Dineen, D.; Gibson, T.J.; Karplus, K.; Li, W.; Lopez, R.; McWilliam, H.; Remmert, M.; Soding, J.; et al. Fast, scalable generation of high-quality protein multiple sequence alignments using Clustal Omega. Mol. Syst. Biol. 2011, 7, 539. [CrossRef]

57. Li, Z.; Guo, Q.; Zheng, L.; Ji, Y.; Xie, Y.T.; Lai, D.H.; Lun, Z.R.; Suo, X.; Gao, N. Cryo-EM structures of the $80 S$ ribosomes from human parasites Trichomonas vaginalis and Toxoplasma gondii. Cell Res. 2017, 27, 1275-1288. [CrossRef] [PubMed]

58. Drinkwater, N.; Vinh, N.B.; Mistry, S.N.; Bamert, R.S.; Ruggeri, C.; Holleran, J.P.; Loganathan, S.; Paiardini, A.; Charman, S.A.; Powell, A.K.; et al. Potent dual inhibitors of Plasmodium falciparum M1 and M17 aminopeptidases through optimization of S1 pocket interactions. Eur. J. Med. Chem. 2016, 110, $43-64$. [CrossRef] [PubMed]

59. Eswar, N.; Webb, B.; Marti-Renom, M.A.; Madhusudhan, M.S.; Eramian, D.; Shen, M.Y.; Pieper, U.; Sali, A. Comparative protein structure modeling using MODELLER. Curr. Protoc. Protein Sci. 2007, 50, 1-4. [CrossRef] [PubMed]

60. Gupta, R.S.; Nanda, A.; Khadka, B. Novel molecular, structural and evolutionary characteristics of the phosphoketolases from bifidobacteria and Coriobacteriales. PLoS ONE 2017, 12. [CrossRef] [PubMed]

61. Kedzierski, L.; Escalante, A.A.; Isea, R.; Black, C.G.; Barnwell, J.W.; Coppel, R.L. Phylogenetic analysis of the genus Plasmodium based on the gene encoding adenylosuccinate lyase. Infect. Genet. Evol. 2002, 1, $297-301$. [CrossRef] 
62. Escalante, A.A.; Barrio, E.; Ayala, F.J. Evolutionary origin of human and primate malarias: Evidence from the circumsporozoite protein gene. Mol. Biol. Evol. 1995, 12, 616-626. [PubMed]

63. Imamura, S.; Yabu, T.; Yamashita, M. Protective role of cell division cycle 48 (CDC48) protein against neurodegeneration via ubiquitin-proteasome system dysfunction during zebrafish development. J. Biol. Chem. 2012, 287, 23047-23056. [CrossRef] [PubMed]

64. McCutchan, T.F.; Kissinger, J.C.; Touray, M.G.; Rogers, M.L.; Li, J.; Sullivan, M.; Braga, E.M.; Krettli, A.U.; Miller, L.H. Comparison of circumsporozoite proteins from avian and mammalian malarias: Biological and phylogenetic implications. Proc. Natl. Acad. Sci. USA 1996, 93, 11889-11894. [CrossRef]

65. Khadka, B.; Gupta, R.S. Identification of a conserved 8 aa insert in the PIP5K protein in the Saccharomycetaceae family of fungi and the molecular dynamics simulations and structural analysis to investigate its potential functional role. Proteins 2017, 85, 1454-1467. [CrossRef]

66. Akiva, E.; Itzhaki, Z.; Margalit, H. Built-in loops allow versatility in domain-domain interactions: Lessons from self-interacting domains. Proc. Natl. Acad. Sci. USA 2008, 105, 13292-13297. [CrossRef]

67. Hashimoto, K.; Panchenko, A.R. Mechanisms of protein oligomerization, the critical role of insertions and deletions in maintaining different oligomeric states. Proc. Natl. Acad. Sci. USA 2010, 107, 20352-20357. [CrossRef] [PubMed]

68. Alonso, P.; Noor, A.M. The global fight against malaria is at crossroads. Lancet 2017, 390, $2532-2534$. [CrossRef]

69. Cox, F.E. History of the discovery of the malaria parasites and their vectors. Parasit. Vectors 2010, 3, 5. [CrossRef] [PubMed]

70. Felsenstein, J. Phylogenies from molecular sequences: Inference and reliability. Annu. Rev. Genet. 1988, 22, 521-565. [CrossRef] [PubMed]

71. Morgan, C.C.; Creevey, C.J.; O'Connell, M.J. Mitochondrial data are not suitable for resolving placental mammal phylogeny. Mamm. Genome 2014, 25, 636-647. [CrossRef] [PubMed]

72. Qari, S.H.; Shi, Y.P.; Pieniazek, N.J.; Collins, W.E.; Lal, A.A. Phylogenetic relationship among the malaria parasites based on small subunit rRNA gene sequences: Monophyletic nature of the human malaria parasite, Plasmodium falciparum. Mol. Phylogenet. Evol. 1996, 6, 157-165. [CrossRef]

73. Escalante, A.A.; Freeland, D.E.; Collins, W.E.; Lal, A.A. The evolution of primate malaria parasites based on the gene encoding cytochrome b from the linear mitochondrial genome. Proc. Natl. Acad. Sci. USA 1998, 95, 8124-8129. [CrossRef]

74. Krief, S.; Escalante, A.A.; Pacheco, M.A.; Mugisha, L.; Andre, C.; Halbwax, M.; Fischer, A.; Krief, J.M.; Kasenene, J.M.; Crandfield, M.; et al. On the diversity of malaria parasites in African apes and the origin of Plasmodium falciparum from Bonobos. PLoS Pathog. 2010, 6. [CrossRef]

75. Kokwaro, G. Ongoing challenges in the management of malaria. Malar. J. 2009, 8, S2. [CrossRef]

76. Mathison, B.A.; Pritt, B.S. Update on Malaria Diagnostics and Test Utilization. J. Clin. Microbiol. 2017, 55, 2009-2017. [CrossRef]

77. Ahmod, N.Z.; Gupta, R.S.; Shah, H.N. Identification of a Bacillus anthracis specific indel in the yeaC gene and development of a rapid pyrosequencing assay for distinguishing B. anthracis from the B. cereus group. J. Microbiol. Methods 2011, 87, 278-285. [CrossRef] [PubMed]

78. Wong, S.Y.; Paschos, A.; Gupta, R.S.; Schellhorn, H.E. Insertion/deletion-based approach for the detection of Escherichia coli O157:H7 in freshwater environments. Environ. Sci. Technol. 2014, 48, 11462-11470. [CrossRef] [PubMed]

79. Hassan, F.M.N.; Gupta, R.S. Novel Sequence Features of DNA Repair Genes/Proteins from Deinococcus Species Implicated in Protection from Oxidatively Generated Damage. Genes 2018, 9, 149. [CrossRef] [PubMed]

80. Singh, B.; Gupta, R.S. Conserved inserts in the Hsp60 (GroEL) and Hsp70 (DnaK) proteins are essential for cellular growth. Mol. Genet. Genom. 2009, 281, 361-373. [CrossRef] [PubMed]

81. Miotto, O.; Almagro-Garcia, J.; Manske, M.; Macinnis, B.; Campino, S.; Rockett, K.A.; Amaratunga, C.; Lim, P.; Suon, S.; Sreng, S.; et al. Multiple populations of artemisinin-resistant Plasmodium falciparum in Cambodia. Nat. Genet. 2013, 45, 648-655. [CrossRef] [PubMed]

82. Hemming-Schroeder, E.; Umukoro, E.; Lo, E.; Fung, B.; Tomas-Domingo, P.; Zhou, G.; Zhong, D.; Dixit, A.; Atieli, H.; Githeko, A.; et al. Impacts of Antimalarial Drugs on Plasmodium falciparum Drug Resistance Markers, Western Kenya, 2003-2015. Am. J. Trop. Med. Hyg. 2018, 98, 692-699. [CrossRef] [PubMed] 
83. Gupta, R.S. Impact of Genomics on Clarifying the Evolutionary Relationships amongst Mycobacteria: Identification of Molecular Signatures Specific for the Tuberculosis-Complex of Bacteria with Potential Applications for Novel Diagnostics and Therapeutics. High Throughput 2018, 7, 31. [CrossRef]

84. Cherkasov, A.; Nandan, D.; Reiner, N.E. Selective targeting of indel-inferred differences in spatial structures of highly homologous proteins. Proteins 2005, 58, 950-954. [CrossRef] 